\title{
Degrees of strongly special subvarieties and the André-Oort conjecture
}

\section{Article}

Published Version

Daw, C. (2016) Degrees of strongly special subvarieties and the André-Oort conjecture. Journal für die reine und angewandte Mathematik (Crelles Journal), 2016 (721). pp. 81108. ISSN 0075-4102 doi: https://doi.org/10.1515/crelle-20140062 Available at https://centaur.reading.ac.uk/70360/

It is advisable to refer to the publisher's version if you intend to cite from the work. See Guidance on citing.

Published version at: http://dx.doi.org/10.1515/crelle-2014-0062

To link to this article DOI: http://dx.doi.org/10.1515/crelle-2014-0062

Publisher: De Gruyter

All outputs in CentAUR are protected by Intellectual Property Rights law, including copyright law. Copyright and IPR is retained by the creators or other copyright holders. Terms and conditions for use of this material are defined in the End User Agreement.

\section{www.reading.ac.uk/centaur}

\section{CentAUR}

Central Archive at the University of Reading

Reading's research outputs online 


\section{Degrees of strongly special subvarieties and the André-Oort conjecture}

Article

Published Version

Degrees of strongly special subvarieties and the Andre-Oort conjecture

Daw, C. (2016) Degrees of strongly special subvarieties and the André-Oort conjecture. Journal für die reine und angewandte Mathematik (Crelles Journal), 2016 (721). pp. 81 108. ISSN 0075-4102 doi: 10.1515/crelle-2014-0062 Available at http://centaur.reading.ac.uk/70360/

It is advisable to refer to the publisher's version if you intend to cite from the work.

Published version at: http://dx.doi.org/10.1515/crelle-2014-0062

To link to this article DOI: http://dx.doi.org/10.1515/crelle-2014-0062

Publisher: De Gruyter

All outputs in CentAUR are protected by Intellectual Property Rights law, including copyright law. Copyright and IPR is retained by the creators or other copyright holders. Terms and conditions for use of this material are defined in the End User Agreement.

www.reading.ac.uk/centaur

\section{CentAUR}

Central Archive at the University of Reading 
Reading's research outputs online 


\title{
Degrees of strongly special subvarieties and the André-Oort conjecture
}

\author{
By Christopher Daw at London
}

\begin{abstract}
In this paper we give a new proof of the André-Oort conjecture under the generalised Riemann hypothesis. In fact, we generalise the strategy pioneered by Edixhoven, and implemented by Klingler and Yafaev, to all special subvarieties. Thus, we remove ergodic theory from the proof of Klingler, Ullmo and Yafaev and replace it with tools from algebraic geometry. Our key ingredient is a lower bound for the degrees of strongly special subvarieties coming from Prasad's volume formula for S-arithmetic quotients of semisimple groups.
\end{abstract}

\section{Introduction}

Definition 1.1. Given a set $\Sigma$ of special subvarieties of a Shimura variety $S$, we denote by $\Sigma$ the subset $\bigcup_{V \in \Sigma} V$ of $S$.

This paper is concerned with the following conjecture:

Conjecture 1.2 (André-Oort). Let $S$ be a Shimura variety and let $\Sigma$ be a set of special points in $S$. Every irreducible component of the Zariski closure of $\Sigma$ in $S$ is a special subvariety.

For the definition of Shimura varieties, special points and special subvarieties we refer the reader to the introduction of [9]. The current paper is complementary to the aforementioned article in the following sense: Klingler and Yafaev consider the above conjecture with $\Sigma$ replaced by a set of special subvarieties, rather than just points. Via extra machinery developed by Ullmo and Yafaev [19], the authors prove the conjecture, assuming the generalised Riemann hypothesis (GRH), by repeatedly replacing the elements of $\Sigma$ with higher dimensional special subvarieties. They rely on a lower bound, obtained by Ullmo and Yafaev, on the degree of the Galois orbit of a special subvariety. As one ranges through the elements of $\Sigma$, this bound is either bounded from above or tends to infinity. In the case that it tends to infinity, the authors are able to proceed using their generalisation of a method pioneered by Edixhoven that compares Galois orbits and Hecke correspondences. Though technical, the proof relies on a simple geometric idea. 
In the case that the lower bound is bounded for all elements in $\Sigma$, Klingler and Yafaev appeal to a result by Ullmo and Yafaev [19], generalising the equidistribution of strongly special subvarieties demonstrated by Clozel and Ullmo [4]. Our motivation was to remove this element of the proof, thus eliminating the dependency on the extremely deep and complicated theorems of Ratner. In this paper, we achieve this aim, thus reproving the following theorem of Clozel and Ullmo:

Theorem 1.3. Let $Z$ be a subvariety of a Shimura variety $S$. There exists a finite set $\left\{V_{1}, \ldots, V_{k}\right\}$ of positive dimensional, strongly special subvarieties $V_{i} \subset Z$ such that, if $V \subset Z$ is a positive dimensional, strongly special subvariety, then $V \subset V_{i}$ for some $i \in\{1, \ldots, k\}$.

Therefore, under the GRH, we are able to prove the André-Oort conjecture solely via the geometric strategy of Edixhoven. In fact, the case dealt with here is less technical and does not depend on the GRH. We employ similar tools from algebraic geometry and the theory of reductive groups over local fields. The main ingredient is the following lower bound for the degrees of strongly special subvarieties. We refer the reader to Sections 2, 3, 4 and 6 for the relevant definitions and explanations.

Theorem 1.4. Let $(G, X)$ be a Shimura datum such that $G=G^{\mathrm{ad}}$ and fix a connected component $X^{+}$of $X$. Fix a faithful representation

$$
\rho: G \hookrightarrow \mathrm{GL}_{n}
$$

and let $K$ be a neat compact open subgroup of $G\left(\mathbb{A}_{f}\right)$ such that $K$ is the product of compact open subgroups $K_{p} \subset G\left(\mathbb{Q}_{p}\right)$. There exist positive constants $c$ and $\delta$ such that, if $V$ is a strongly special subvariety of $S_{K}(G, X)$, defined by $\left(H, X_{H}\right)$, then

$$
\operatorname{deg}_{\mathscr{L}_{K}} V>c \cdot \Pi\left(H, K_{H}\right)^{\delta} .
$$

This bound replaces the lower bound on the degrees of Galois orbits used in [9]. Otherwise, the strategy is largely similar, though somewhat simplified in this case since we will not need an analogue of [9, Lemma 9.2.3]. Given a strongly special subvariety $V$, contained in an irreducible subvariety $Z$, one obtains a lower bound for the degree of $V$ in terms of a product of 'bad' primes (see Theorem 1.4). One then obtains a 'good' prime $p$, small compared to the degree of $V$, such that there exists a 'suitable' Hecke correspondence $T$ at $p$ satisfying $V \subset T(V)$. Thus, $V$ is contained in the intersection $Z \cap T(Z)$. However, if the dimension of $Z$ is only one greater than that of $V$, comparing their degrees leads one to realise that the intersection $Z \cap T(Z)$ cannot be proper. Therefore, since $Z$ is irreducible, it must be contained in $T(Z)$. In this case, a geometric argument implies that there exists a strongly special subvariety $V^{\prime} \subset Z$ such that $V \subsetneq V^{\prime}$. On the other hand, if the intersection $Z \cap T(Z)$ is proper, one chooses an irreducible component containing $V$ and repeats the above procedure.

This result represents the full generalisation of a strategy tested in [5] for removing ergodic theory from the proof of the André-Oort conjecture. However, we also hope that the bounds presented here will lead to useful developments in the wider world of the Zilber-Pink conjectures. 


\section{Generalities}

Unless stated otherwise, all varieties (except for linear algebraic groups) will be defined over $\mathbb{C}$ and identified with their set of $\mathbb{C}$-points. We will denote by $\mathbb{A}_{f}$ the ring of finite (rational) adeles and by $\widehat{\mathbb{Z}}$ the product of $\mathbb{Z}_{p}$ over all primes $p$.

For any algebraic group $G$, we will denote by $G^{\text {ad }}$ the quotient of $G$ by its centre. If $G$ is defined over $\mathbb{Q}_{p}$ and $\rho$ is a faithful representation, we will consider $G$ as a subgroup of $\mathrm{GL}_{n}, \mathbb{Q}_{p}$. For such a subgroup, we will denote by $G_{\mathbb{Z}_{p}}$ the Zariski closure of $G$ in $\mathrm{GL}_{n}, \mathbb{Z}_{p}$. We will say that $G$ is unramified if it is quasi-split and splits over an unramified extension of $\mathbb{Q}_{p}$. If $G$ and $\rho$ are defined over $\mathbb{Q}$, then the previous definitions make sense for $G_{\mathbb{Q}_{p}}$ and $\rho_{\mathbb{Q}_{p}}$ for any prime $p$. A subgroup $K_{p} \subset G\left(\mathbb{Q}_{p}\right)$ is called hyperspecial if there exists a smooth reductive group scheme $\mathbf{G}$ over $\mathbb{Z}_{p}$ such that $\mathbf{G}_{\mathbb{Q}_{p}}=G_{\mathbb{Q}_{p}}$ and $\mathbf{G}\left(\mathbb{Z}_{p}\right)=K_{p}$ (see [2, Section 4.6]). By a reductive group scheme, we mean a group scheme with reductive fibres.

Given an algebraic torus $T$ over a field $k$ and a representation

$$
\rho: T \hookrightarrow \mathrm{GL}_{n, k},
$$

let $l / k$ be a Galois extension such that $T_{l}$ splits. One obtains a decomposition $l^{n}=\bigoplus_{\chi} V_{\chi}$, summing over characters $\chi: T_{l} \rightarrow \mathbb{G}_{m, l}$ of $T$, where $V_{\chi}$ is the $l$-subspace on which $T_{l}$ acts via $\chi$. We refer to those characters $\chi$ such that $V_{\chi} \neq\{0\}$ as the characters intervening in $l^{n}$. The characters of $T$ form a free $\mathbb{Z}$-module $X^{*}(T)$ equipped with an action of $\operatorname{Gal}(l / k)$. After choosing a basis for $X^{*}(T)$, one may refer to the coordinates of a character $\chi \in X^{*}(T)$.

Let $X$ be a complete irreducible variety and let $\mathscr{L}$ be a line bundle on $X$ with topological first Chern class

$$
c_{1}(\mathscr{L}) \in H^{2}(X, \mathbb{Z}) .
$$

Given an irreducible subvariety $V$ of $X$, we define the degree of $V$, with respect to $\mathscr{L}$, as in $[9$, Section 5.1], by

$$
\operatorname{deg}_{\mathscr{L}} V:=c_{1}(\mathscr{L})^{\operatorname{dim} V} \cap[V] \in H_{0}(X, \mathbb{Z})=\mathbb{Z},
$$

where $[V] \in H_{2 \operatorname{dim} V}(X, \mathbb{Z})$ denotes the fundamental class of $V$ and $\cap$ denotes the cap product between $H^{2 \operatorname{dim} V}(X, \mathbb{Z})$ and $H_{2 \operatorname{dim} V}(X, \mathbb{Z})$. We will also put

$$
\int_{V} c_{1}(\mathscr{L})^{\operatorname{dim} V}:=\operatorname{deg}_{\mathscr{L}} V
$$

When the variety $X$ is a disjoint union of irreducible components $X_{i}$, the function $\operatorname{deg}_{\mathscr{L}}$ is defined as the $\operatorname{sum} \sum_{i} \operatorname{deg}_{\mathscr{L} \mid X_{i}}$.

\section{Reductions}

Consider a Shimura datum $(G, X)$, a connected component $X^{+}$of $X$, and a compact open subgroup $K$ of $G\left(\mathbb{A}_{f}\right)$. We will write $\operatorname{Sh}_{K}(G, X)$ for the corresponding Shimura variety and denote by $S_{K}(G, X)$ the image of $X^{+} \times\{1\}$ in $\operatorname{Sh}_{K}(G, X)$. Recall that the André-Oort conjecture is equivalent for all choices of $K$.

We will write $\overline{\operatorname{Sh}_{K}(G, X)}$ for the Baily-Borel compactification of $\operatorname{Sh}_{K}(G, X)$ (as defined in [9, Proposition 5.3.1]) and $\mathscr{L}_{K}$ for the corresponding ample line bundle (as defined in [9, Proposition 5.3.2])). For an irreducible subvariety $V$ of $\operatorname{Sh}_{K}(G, X)$ we will denote by $\bar{V}$ the Zariski closure of $V$ in $\overline{\operatorname{Sh}_{K}(G, X)}$. We will write $\operatorname{deg}_{\mathscr{L}_{K}} V$ for $\operatorname{deg}_{\mathscr{L}_{K}} \bar{V}$. 
We denote by $X_{\text {ad }}$ the $G^{\text {ad }}(\mathbb{R})$-conjugacy class of morphisms from $\mathbb{S}:=\operatorname{Res}_{\mathbb{C} / \mathbb{R}} \mathbb{G}_{m}, \mathbb{C}$ to $G_{\mathbb{R}}^{\text {ad }}$ that contains the image of $X$. Then $\left(G^{\text {ad }}, X_{\text {ad }}\right)$ is a Shimura datum, referred to as the adjoint Shimura datum. The image $K_{\mathrm{ad}}$ of $K$ in $G^{\mathrm{ad}}\left(\mathbb{A}_{f}\right)$ is a compact open subgroup and we have an induced morphism

$$
\operatorname{Sh}_{K}(G, X) \rightarrow \operatorname{Sh}_{K_{\text {ad }}}\left(G^{\mathrm{ad}}, X_{\mathrm{ad}}\right) .
$$

By [8, Proposition 2.2], $V$ is special if and only if its image $V_{\text {ad }}$ is special. Hence, for the purposes of the André-Oort conjecture, we may assume that $G=G^{\mathrm{ad}}$.

Let $\alpha \in G\left(\mathbb{A}_{f}\right)$ and let $T_{\alpha}$ be the associated Hecke correspondence on $\operatorname{Sh}_{K}(G, X)$. By the definition of a special subvariety, $V$ is special if and only if one (or, equivalently, all) of the irreducible components of $T_{\alpha}(V)$ is (are) special. In particular, in order to prove the André-Oort conjecture, it suffices to consider sets of special subvarieties $\Sigma$ such that the Zariski closure of $\Sigma$ in $\operatorname{Sh}_{K}(G, X)$ is irreducible and contained in $S_{K}(G, X)$.

In this paper, we will often have an inclusion of Shimura data $\left(G_{1}, X_{1}\right) \subset\left(G_{2}, X_{2}\right)$ and a compact open subgroup $K_{1}:=K_{2} \cap G_{1}\left(\mathbb{A}_{f}\right)$ of $G_{1}\left(\mathbb{A}_{f}\right)$, where $K_{2}$ is a compact open subgroup of $G_{2}\left(\mathbb{A}_{f}\right)$. Thus, we obtain a morphism

$$
\phi: \operatorname{Sh}_{K_{1}}\left(G_{1}, X_{1}\right) \rightarrow \operatorname{Sh}_{K_{2}}\left(G_{2}, X_{2}\right)
$$

and, by [19, Lemma 2.2], if $K_{2}$ is neat, $\phi$ is generically injective. In this case, we will use the same symbol for a subvariety of $\operatorname{Sh}_{K_{1}}\left(G_{1}, X_{1}\right)$ and its image in $\operatorname{Sh}_{K_{2}}\left(G_{2}, X_{2}\right)$.

\section{Choosing a measure}

Consider a special subvariety $V$ of $S_{K}(G, X)$. By [19, Lemma 2.1], there exists a Shimura subdatum $\left(H, X_{H}\right)$ of $(G, X)$ and a connected component $X_{H}^{+}$of $X_{H}$ contained in $X^{+}$ such that $H$ is the generic Mumford-Tate group on $X_{H}$ and $V$ is the image of $X_{H}^{+} \times\{1\}$ in $\operatorname{Sh}_{K}(G, X)$. We will denote by $K_{H}$ the intersection $K \cap H\left(\mathbb{A}_{f}\right)$ and by $\Gamma_{H}$ the intersection $H(\mathbb{Q})_{+} \cap K_{H}$, where $H(\mathbb{Q})_{+}$is the stabiliser of $X_{H}^{+}$in $H(\mathbb{Q})$. Thus, $V$ is the image of $\Gamma_{H} \backslash X_{H}^{+}$in $\operatorname{Sh}_{K}(G, X)$. We refer to $\left(H, X_{H}\right)$ as the Shimura datum defining $V$ and we say that $V$ is strongly special if the image of $H$ in $G^{\text {ad }}$ is semisimple.

The space $X_{H}^{+}$is isomorphic to $H^{\text {ad }}(\mathbb{R})^{+} / K_{\infty}$, where $K_{\infty}$ is a maximal compact subgroup of $H^{\text {ad }}(\mathbb{R})^{+}$. Let $\mathfrak{h}$ denote the Lie algebra of $H^{\text {ad }}(\mathbb{R})^{+}$and let $\mathfrak{h}^{*}$ denote the dual of $\mathfrak{h}$. Any real, non-zero, left-invariant differential form $\omega$ of maximal degree $r$ on $H^{\text {ad }}(\mathbb{R})^{+}$ corresponds to an element of $\bigwedge^{r} \mathfrak{h}^{*}$.

Since $\mathfrak{h}$ admits a Cartan decomposition $\mathfrak{k} \oplus \mathfrak{p}$, where $\mathfrak{k}$ is the Lie algebra of $K_{\infty}$ and $\mathfrak{p}$ is the tangent space of $X_{H}^{+}$at the point $K_{\infty}$, we can write $\omega=\omega_{\mathfrak{k}} \wedge \omega_{\mathfrak{p}}$, where $\omega_{\mathfrak{k}}$ and $\omega_{\mathfrak{p}}$ correspond to real multilinear forms on $\mathfrak{k}$ and $\mathfrak{p}$, respectively. In this paper, we will always choose $\omega_{\mathfrak{F}}$ so that, with respect to the measure it determines, the volume of $K_{\infty}$ is one.

Consider the unique (up to isomorphism) $\mathbb{R}$-anisotropic form $H^{c}$ of $H^{\text {ad }}$, i.e. the real algebraic group $H^{c}$ isomorphic to $H^{\text {ad }}$ over $\mathbb{C}$ such that $H^{c}(\mathbb{R})$ is compact. Then $H^{c}(\mathbb{R})$ is a connected, maximal, compact subgroup of $H^{c}(\mathbb{C})$ containing a copy of $K_{\infty}$ and the quotient $\check{X}_{H}:=H^{c}(\mathbb{R}) / K_{\infty}$ is called the compact dual of $X_{H}^{+}$. It contains $X_{H}^{+}$as an open subset.

Considering multilinear forms on the complexification $\mathfrak{h} \mathbb{C}:=\mathfrak{h} \otimes \mathbb{C}$, the form $\omega$ extends $\mathbb{C}$-linearly to a complex, left-invariant differential form $\omega_{\mathbb{C}}$ on $H^{\text {ad }}(\mathbb{C})$. As in [13, Proportion-

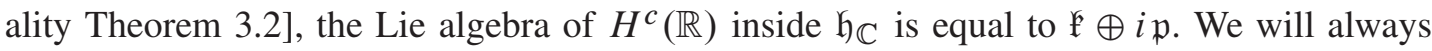


choose $\omega_{\mathfrak{p}}$ so that, with respect to the measure determined by $\omega_{\mathbb{C}}$, the volume of $H^{c}(\mathbb{R})$ or, equivalently, any maximal compact subgroup of $H^{\text {ad }}(\mathbb{C})$, is one. Therefore, the volume of $\check{X}_{H}$ is also one.

We will denote by $\mu$ the Haar measure on $H^{\text {ad }}(\mathbb{R})^{+}$determined by $\omega$. We will also denote by $\mu$ the volume measure on $X_{H}^{+}$determined by $\omega_{\mathfrak{p}}$. When we consider the volume measures induced on arithmetic quotients of either $H^{\text {ad }}(\mathbb{R})^{+}$or $X_{H}^{+}$, we will again use $\mu$.

\section{Degrees of strongly special subvarieties}

In order to prove Theorem 1.4, we will prove the following theorem, relating the degree of a special subvariety to its volume:

Theorem 5.1. Let $(G, X)$ be a Shimura datum, let $X^{+}$be a connected component of $X$, and let $K$ be a neat compact open subgroup of $G\left(\mathbb{A}_{f}\right)$. There exists a constant $c_{1}$ such that, if $V$ is a special subvariety of $S_{K}(G, X)$, defined by $\left(H, X_{H}\right)$, then

$$
\operatorname{deg}_{\mathscr{L}_{K}} V>c_{1} \cdot \mu\left(\Gamma_{H} \backslash X_{H}^{+}\right) .
$$

In this paper, a constant will be taken to mean a positive real number.

Proof. By [9, Corollary 5.3.10],

$$
\operatorname{deg}_{\mathscr{L}_{K}} V \geq \operatorname{deg}_{\mathscr{L}_{K_{H}}} V
$$

and, for the remainder of this section, $V$ will refer to the connected component $\Gamma_{H} \backslash X_{H}^{+}$ of $\operatorname{Sh}_{K_{H}}\left(H, X_{H}\right)$.

Consider a smooth compactification $\bar{V}^{\text {sm }}$ of $V$, thus providing a canonical birational map

$$
\pi: \bar{V}^{\mathrm{sm}} \rightarrow \bar{V}
$$

as in the proof of [13, Proposition 3.4(b)]. By [9, Proposition 5.3.2(1)], the exterior product $\Omega^{\operatorname{dim} X_{H}}$ of the cotangent bundle $\Omega$ on $X_{H}$ descends to $\operatorname{Sh}_{K_{H}}\left(H, X_{H}\right)$ and extends uniquely to an ample line bundle on $\bar{V}$ (this is the restriction of $\mathscr{L}_{K_{H}}$ ). By [13, Proposition 3.4 (b)], the pullback $\pi^{*} \mathscr{L}_{K_{H}}$ of $\mathscr{L}_{K_{H}}$ to $\bar{V}^{\mathrm{sm}}$ is the unique extension $\bar{E}$ of [13, Main Theorem 3.1]. Of course, $\Omega^{\operatorname{dim} X_{H}}$ is the restriction of the exterior product $\check{\Omega}^{\operatorname{dim} X_{H}}$ of the cotangent bundle $\check{\Omega}$ on the compact dual $\check{X}_{H}$. By [13, Proportionality Theorem 3.2], we have

$$
\operatorname{deg}_{\pi^{*} \mathscr{L}_{K_{H}}} \bar{V}^{\mathrm{sm}}=(-1)^{\operatorname{dim} X_{H}} \cdot \mu\left(\Gamma_{H} \backslash X_{H}^{+}\right) \cdot \int_{\check{X}_{H}} c_{1}\left(\check{\Omega}^{\operatorname{dim} X_{H}}\right)^{\operatorname{dim} X_{H}} .
$$

However, since $\pi$ is birational, the projection formula (see [9, Section 5.1]) implies that

$$
\operatorname{deg}_{\pi^{*} \mathscr{L}_{K_{H}}} \bar{V}^{\mathrm{sm}}=\operatorname{deg}_{\mathscr{L}_{K_{H}}} V .
$$

Furthermore, up to isomorphism, the number of Hermitian symmetric spaces corresponding to Shimura subdata of $(G, X)$ is finite. Therefore,

$$
(-1)^{\operatorname{dim} X_{H}} \cdot \int_{\check{X}_{H}^{+}} c_{1}\left(\check{\Omega}^{\operatorname{dim} X_{H}}\right)^{\operatorname{dim} X_{H}}
$$

may assume only finitely many positive values. 


\section{Volumes of strongly special subvarieties}

In this section, we prove a lower bound for the volume of a strongly special subvariety, concluding the proof of Theorem 1.4. First, however, suppose that $G$ is a reductive group over $\mathbb{Q}$ and $L$ is a finite Galois extension over which $G$ is split. Since almost all places of $L$ are unramified over $\mathbb{Q}$, it follows that $G_{\mathbb{Q}_{p}}$ is split over an unramified extension for almost all primes $p$. Furthermore, by [17, Lemma 4.9 (ii)], $G_{\mathbb{Q}_{p}}$ is quasi-split for almost all primes $p$. Therefore, we let $\Sigma(G)$ denote the finite set of primes $p$ such that $G_{\mathbb{Q}_{p}}$ is not unramified.

Suppose that $K$ is a compact open subgroup of $G\left(\mathbb{A}_{f}\right)$, equal to a product of compact open subgroups $K_{p} \subset G\left(\mathbb{Q}_{p}\right)$; fix a faithful representation $G \hookrightarrow \mathrm{GL}_{n}$. By [9, Section 4.1.5], $K_{p}=G_{\mathbb{Z}_{p}}\left(\mathbb{Z}_{p}\right)$ for almost all primes $p$. Thus, by [18, Section 3.9.1], $K_{p}$ is hyperspecial for almost all $p$. Therefore, we let $\Sigma(K)$ denote the finite set of primes $p$ such that $K_{p}$ is not hyperspecial. Finally, we let $\Sigma(G, K)$ denote the set of primes belonging to either $\Sigma(G)$ or $\Sigma(K)$ and we let $\Pi(G, K)$ denote their product.

Theorem 6.1. Let $(G, X)$ be a Shimura datum such that $G=G^{\mathrm{ad}}$ and let $X^{+}$be a connected component of $X$. Fix a faithful representation

$$
\rho: G \hookrightarrow \mathrm{GL}_{n}
$$

and let $K$ be a neat compact open subgroup of $G\left(\mathbb{A}_{f}\right)$, equal to a product of compact open subgroups $K_{p} \subset G\left(\mathbb{Q}_{p}\right)$. There exist constants $c_{2}$ and $\delta$ such that, if $V$ is a strongly special subvariety of $S_{K}(G, X)$, defined by $\left(H, X_{H}\right)$, then

$$
\mu\left(\Gamma_{H} \backslash X_{H}^{+}\right)>c_{2} \cdot \Pi\left(H, K_{H}\right)^{\delta} .
$$

In this section, we will use the term uniform to mean depending only on $(G, X), K$ and $\rho$.

Note that, since $K_{H}$ is neat, $\Gamma_{H}$ injects into $H^{\text {ad }}(\mathbb{R})^{+}$and so acts freely on $X_{H}^{+}$. Let ad : $H \rightarrow H^{\text {ad }}$ denote the natural map. Since $K_{\infty}$ has volume one with respect to the measure determined by $\omega_{\mathfrak{k}}$, we have

$$
\mu\left(\Gamma_{H} \backslash X_{H}^{+}\right)=\mu\left(\operatorname{ad}\left(\Gamma_{H}\right) \backslash H^{\mathrm{ad}}(\mathbb{R})^{+}\right) .
$$

Since $H$ is semisimple, we have a central isogeny $\pi: \widetilde{H} \rightarrow H$, where $\widetilde{H}$ is simply connected and whose centre we denote $Z_{\widetilde{H}}$. We denote by $Z \subset Z_{\widetilde{H}}$ the kernel of $\pi$. Note that, by [10, Proposition 1.4.5], the maximal split tori (resp. parabolic subgroups) of $\widetilde{H}$ are in bijection via this morphism with the maximal split tori (resp. parabolic subgroups) of $H$. Therefore, $\Sigma(\widetilde{H})=\Sigma(H)$.

Since $\pi$ is finite, and therefore proper,

$$
\widetilde{K}_{H}:=\pi_{\mathbb{A}_{f}}^{-1}\left(K_{H}\right)
$$

is a compact open subgroup of $\widetilde{H}\left(\mathbb{A}_{f}\right)$ and we let

$$
\widetilde{\Gamma}_{H}:=\widetilde{H}(\mathbb{Q}) \cap \widetilde{K}_{H},
$$

which is equal to $\pi^{-1}\left(\Gamma_{H}\right)$. Since $K_{H}$ is necessarily a product of compact open subgroups $K_{H, p} \subset H\left(\mathbb{Q}_{p}\right), \widetilde{K}_{H}$ is also a product of compact open subgroups $\widetilde{K}_{H, p} \subset \widetilde{H}\left(\mathbb{Q}_{p}\right)$. Let $K_{\widetilde{H}}^{m}$ be a maximal compact open subgroup of $\widetilde{H}\left(\mathbb{A}_{f}\right)$ containing $\widetilde{K}_{H}$ and let

$$
\Gamma_{\widetilde{H}}^{m}:=\widetilde{H}(\mathbb{Q}) \cap K_{\widetilde{H}}^{m} .
$$

Again $K_{\widetilde{H}}^{m}$ is a product of maximal compact open subgroups $K_{\widetilde{H}, p}^{m} \subset \widetilde{H}\left(\mathbb{Q}_{p}\right)$. 
By [11, Theorem 5.2], $\widetilde{H}(\mathbb{R})$ is connected and so acts on $H^{\text {ad }}(\mathbb{R})^{+}$through ad $\circ \pi$. Therefore, we have two finite projections

$$
\operatorname{ad}\left(\Gamma_{H}\right) \backslash H^{\mathrm{ad}}(\mathbb{R})^{+} \leftarrow \operatorname{ad} \circ \pi\left(\widetilde{\Gamma}_{H}\right) \backslash H^{\mathrm{ad}}(\mathbb{R})^{+} \rightarrow \operatorname{ad} \circ \pi\left(\Gamma_{\widetilde{H}}^{m}\right) \backslash H^{\mathrm{ad}}(\mathbb{R})^{+}
$$

yielding the equality

$$
\mu\left(\operatorname{ad}\left(\Gamma_{H}\right) \backslash H^{\operatorname{ad}}(\mathbb{R})^{+}\right)=\frac{\left[\operatorname{ad} \circ \pi\left(\Gamma_{\widetilde{H}}^{m}\right): \operatorname{ad} \circ \pi\left(\widetilde{\Gamma}_{H}\right)\right]}{\left[\operatorname{ad}\left(\Gamma_{H}\right): \operatorname{ad} \circ \pi\left(\widetilde{\Gamma}_{H}\right)\right]} \cdot \mu\left(\operatorname{ad} \circ \pi\left(\Gamma_{\widetilde{H}}^{m}\right) \backslash H^{\operatorname{ad}}(\mathbb{R})^{+}\right) .
$$

Lemma 6.2. There exists a uniform constant $c_{3}$ such that

$$
\left[\operatorname{ad} \circ \pi\left(\Gamma_{\widetilde{H}}^{m}\right): \operatorname{ad} \circ \pi\left(\widetilde{\Gamma}_{H}\right)\right]>c_{3}\left[K_{\widetilde{H}}^{m}: \widetilde{K}_{H}\right] .
$$

Proof. Consider the surjective map

$$
\Gamma_{\widetilde{H}}^{m} \rightarrow \operatorname{ad} \circ \pi\left(\Gamma_{\widetilde{H}}^{m}\right) / \operatorname{ad} \circ \pi\left(\widetilde{\Gamma}_{H}\right) .
$$

Since $\widetilde{\Gamma}_{H}=\pi^{-1}\left(\Gamma_{H}\right)$, the kernel is equal to

$$
(\operatorname{ad} \circ \pi)^{-1}\left(\operatorname{ad} \circ \pi\left(\pi^{-1}\left(\Gamma_{H}\right)\right)\right) \cap \Gamma_{\widetilde{H}}^{m},
$$

which is readily seen to be $Z_{\widetilde{H}}(\mathbb{Q}) \cdot \pi^{-1}\left(\Gamma_{H}\right)$. Therefore, we turn our attention to $\left[\Gamma_{\widetilde{H}}^{m}: \widetilde{\Gamma}_{H}\right]$. Write

$$
K_{\widetilde{H}}^{m}=\coprod_{i=1}^{n} k_{i} \widetilde{K}_{H}
$$

where $k_{i} \in K_{\widetilde{H}}^{m}$. By strong approximation (as in [11, Theorem 4.16]), applied to $\widetilde{H}$, each $k_{i}$ can be written as $q_{i} k_{i}^{\prime}$, where $q_{i} \in \widetilde{H}(\mathbb{Q})$ and $k_{i}^{\prime} \in \widetilde{K}_{H}$. Therefore, in the above, we may replace $k_{i}$ with $q_{i}$. Intersecting both sides with $\widetilde{H}(\mathbb{Q})$ we obtain

$$
\Gamma_{\widetilde{H}}^{m}=\coprod_{i=1}^{n} q_{i} \widetilde{\Gamma}_{H},
$$

and so $q_{i} \in \Gamma_{\widetilde{H}}^{m}$.

Lemma 6.3. There exist uniform constants $c_{4}$ and $C$ such that

$$
\left[\operatorname{ad}\left(\Gamma_{H}\right): \operatorname{ad} \circ \pi\left(\widetilde{\Gamma}_{H}\right)\right]<c_{4} C^{\left|\Sigma\left(H, K_{H}\right)\right|} .
$$

Proof. Consider the surjective map

$$
\Gamma_{H} \rightarrow \operatorname{ad}\left(\Gamma_{H}\right) / \operatorname{ad} \circ \pi\left(\widetilde{\Gamma}_{H}\right) .
$$

The kernel is equal to

$$
\operatorname{ad}^{-1}\left(\operatorname{ad} \circ \pi\left(\widetilde{\Gamma}_{H}\right)\right) \cap \Gamma_{H},
$$

which is readily seen to be

$$
\left(\pi\left(\widetilde{\Gamma}_{H}\right) \cdot Z_{H}(\mathbb{Q})\right) \cap \Gamma_{H}=\pi\left(\widetilde{\Gamma}_{H}\right) \cdot\left(Z_{H}(\mathbb{Q}) \cap \Gamma_{H}\right),
$$

where $Z_{H}$ is the centre of $H$, whose order is uniformly bounded by the proof of [19, Lemma 2.4]. Therefore, we turn our attention to $\left[\Gamma_{H}: \pi\left(\widetilde{\Gamma}_{H}\right)\right]$. 
Recall that Galois cohomology yields an exact sequence

$$
\widetilde{H}(\mathbb{Q}) \rightarrow H(\mathbb{Q}) \rightarrow H^{1}(\operatorname{Gal}(\overline{\mathbb{Q}} / \mathbb{Q}), Z(\overline{\mathbb{Q}})) .
$$

Therefore, $\pi\left(\widetilde{\Gamma}_{H}\right) \backslash \Gamma_{H}$ embeds as a subgroup of the Abelian group $H^{1}(\operatorname{Gal}(\overline{\mathbb{Q}} / \mathbb{Q}), Z(\overline{\mathbb{Q}}))$. On the other hand, $\pi\left(\widetilde{\Gamma}_{H}\right) \backslash \Gamma_{H}$ embeds into

$$
\pi\left(\widetilde{K}_{H}\right) \backslash K_{H}=\prod_{p} \pi\left(\widetilde{K}_{H, p}\right) \backslash K_{H, p}
$$

and, again, Galois cohomology tells us that, for all primes $p$,

$$
\pi\left(\widetilde{K}_{H, p}\right) \backslash K_{H, p} \hookrightarrow H^{1}\left(\operatorname{Gal}\left(\overline{\mathbb{Q}_{p}} / \mathbb{Q}_{p}\right), Z\left(\overline{\mathbb{Q}_{p}}\right)\right) .
$$

However, now consider a prime $p$ such that $H_{\mathbb{Q}_{p}}$ is unramified and $K_{H, p}$ is hyperspecial. Since $\widetilde{H}_{\mathbb{Q}_{p}}$ is also unramified, it follows that $\widetilde{H}\left(\mathbb{Q}_{p}\right)$ also possesses hyperspecial subgroups by [18, Section 3.8.2]. Therefore, by [18, Section 3.8.1], there exist smooth reductive group schemes $\widetilde{\mathbf{H}}$ and $\mathbf{H}$ over $\mathbb{Z}_{p}$, the generic fibres of which are $\widetilde{H}_{\mathbb{Q}_{p}}$ and $H_{\mathbb{Q}_{p}}$, such that $K_{H, p}=\mathbf{H}\left(\mathbb{Z}_{p}\right)$ and $\widetilde{\mathbf{H}}\left(\mathbb{Z}_{p}\right)$ is a hyperspecial subgroup of $\widetilde{H}\left(\mathbb{Q}_{p}\right)$. By [20, Lemma 2.3.1], the central isogeny $\pi_{\mathbb{Q}_{p}}$ extends uniquely to a central isogeny $\pi_{\mathbb{Z}_{p}}: \widetilde{\mathbf{H}} \rightarrow \mathbf{H}$. Therefore, the kernel $\mathbf{Z}$ of $\pi_{\mathbb{Z}_{p}}$ is a finite group scheme of multiplicative type such that $\mathbf{Z}_{\mathbb{Q}_{p}}=Z_{\mathbb{Q}_{p}}$. Over a finite Galois extension $F$ of $\mathbb{Q}, Z_{F}$ is isomorphic to a product of roots of unity, whose orders we denote $n_{1}, \ldots, n_{r}$. Therefore, by [7, Exposé X, Lemme 4.1], if $p$ is coprime to the $n_{i}$, $\mathbf{Z}_{\mathbb{F}_{p}}$ is smooth.

Therefore, by [15, Lemma 6.5], for any prime $p \notin \Sigma\left(H, K_{H}\right)$ and coprime to the $n_{i}$, we have an exact sequence

$$
\widetilde{\mathbf{H}}\left(\mathbb{Z}_{p}\right) \rightarrow \mathbf{H}\left(\mathbb{Z}_{p}\right) \rightarrow H^{1}\left(\operatorname{Gal}\left(\mathbb{Q}_{p}^{\text {un }} / \mathbb{Q}_{p}\right), \mathbf{Z}\left(\mathbb{Z}_{p}^{\text {un }}\right)\right),
$$

where $\mathbb{Q}_{p}^{\text {un }}$ is the maximal unramified extension of $\mathbb{Q}_{p}$ and $\mathbb{Z}_{p}^{\text {un }}$ is its ring of integers. However, since $\widetilde{K}_{H, p}$ clearly contains $\widetilde{\mathbf{H}}\left(\mathbb{Z}_{p}\right)$ and, by [18, Section 3.8 .2$], \widetilde{\mathbf{H}}\left(\mathbb{Z}_{p}\right)$ is maximal among compact subgroups of $\widetilde{H}\left(\mathbb{Q}_{p}\right)$, we have $\widetilde{\mathbf{H}}\left(\mathbb{Z}_{p}\right)=\widetilde{K}_{H, p}$.

By $[6$, Theorem 5.1], the degree $[F: \mathbb{Q}]$ is bounded in terms of the dimension of any maximal torus of $\widetilde{H}$ containing $Z$, which is itself bounded by a uniform constant. By the proof of [19, Lemma 2.4], the order of $Z$ is also bounded by a uniform constant and so the same can be said of $\left|H^{1}(\operatorname{Gal}(F / \mathbb{Q}), Z(F))\right|$. Therefore, we may consider the image of the quotient $\pi\left(\widetilde{\Gamma}_{H}\right) \backslash \Gamma_{H}$ in $H^{1}(\operatorname{Gal}(\overline{\mathbb{Q}} / F), Z(\overline{\mathbb{Q}}))$, whose image in $H^{1}\left(\operatorname{Gal}\left(\overline{\mathbb{Q}_{p}} / F_{v}\right), Z\left(\overline{\mathbb{Q}_{p}}\right)\right)$ is contained in $H^{1}\left(\operatorname{Gal}\left(\mathbb{Q}_{p}^{\text {un }} / F_{v}\right), \mathbf{Z}\left(\mathbb{Z}_{p}^{\text {un }}\right)\right)$ for all places $v$ of $F$ lying above a prime $p \notin \Sigma\left(H, K_{H}\right)$ coprime to the $n_{i}$.

We identify the three previous cohomology groups with the groups

$$
\prod_{i=1}^{r}\left(F^{*}\right)^{n_{i}} \backslash F^{*}, \quad \prod_{i=1}^{r}\left(F_{v}^{*}\right)^{n_{i}} \backslash F_{v}^{*} \quad \text { and } \quad \prod_{i=1}^{r}\left(\mathcal{O}_{F_{v}}^{*}\right)^{n_{i}} \backslash \mathcal{O}_{F_{v}}^{*}
$$

and choose a uniformiser $\xi_{v} \in F$ at each place $v$ lying above a prime $p \in \Sigma\left(H, K_{H}\right)$ or dividing one of the $n_{i}$. Therefore, the image of

$$
\pi\left(\widetilde{\Gamma}_{H}\right) \backslash \Gamma_{H} \rightarrow \prod_{i=1}^{r}\left(F^{*}\right)^{n_{i}} \backslash F^{*}
$$

is contained in the subgroup generated by $\mathcal{O}_{F}^{*}$ and the $\xi_{v}$. Now, $\mathcal{O}_{F}^{*}$ is a finitely generated Abelian group whose rank and torsion subgroup are uniformly bounded and, since the $n_{i}$ are bounded by the order of $Z$, we are done. 
We now appeal to Prasad's formula.

Lemma 6.4. There exist uniform constants $c_{5}$ and $\delta_{1}$ such that

$$
\mu\left(\operatorname{ad} \circ \pi\left(\Gamma_{\widetilde{H}}^{m}\right) \backslash H^{\mathrm{ad}}(\mathbb{R})^{+}\right)>c_{5} \cdot \Pi\left(\widetilde{H}, K_{\widetilde{H}}^{m}\right)^{\delta_{1}} .
$$

Proof. Let

$$
\widetilde{\omega}:=\frac{1}{\left|Z_{\widetilde{H}}\right|} \omega^{*},
$$

where $\omega^{*}$ is the pullback of $\omega$ to $\widetilde{H}(\mathbb{R})$. Denote by $\widetilde{\mu}$ the measure determined by $\widetilde{\omega}$ on $\widetilde{H}(\mathbb{R})$ and its arithmetic quotients. By [11, Proposition 5.1], $\widetilde{H}(\mathbb{R}) \rightarrow H^{\text {ad }}(\mathbb{R})^{+}$is surjective. On the other hand, the kernel of the map

$$
\Gamma_{\widetilde{H}}^{m} \backslash \widetilde{H}(\mathbb{R}) \rightarrow \operatorname{ad} \circ \pi\left(\Gamma_{\widetilde{H}}^{m}\right) \backslash H^{\mathrm{ad}}(\mathbb{R})^{+}
$$

is $\Gamma_{\widetilde{H}}^{m} \cap Z_{\widetilde{H}}(\mathbb{R}) \backslash Z_{\widetilde{H}}(\mathbb{R})$. It follows from the proof of [19, Lemma 2.4] that

$$
\frac{\mu\left(\operatorname{ad} \circ \pi\left(\Gamma_{\widetilde{H}}^{m}\right) \backslash H^{\mathrm{ad}}(\mathbb{R})^{+}\right)}{\widetilde{\mu}\left(\Gamma_{\widetilde{H}}^{m} \backslash \widetilde{H}(\mathbb{R})\right)}
$$

is greater than a uniform constant.

Since $\widetilde{H}$ is simply connected, it is a direct product $H_{1} \times \cdots \times H_{s}$ of quasi-simple, simply connected subgroups. We can write $\widetilde{\omega}=\omega_{1} \wedge \cdots \wedge \omega_{s}$, where $\omega_{i}$ is a real, non-zero, left-invariant differential form of maximal degree on $H_{i}(\mathbb{R})$. Since ad $\circ \pi$ is surjective, of degree $\left|Z^{\prime}\right|$ and proper, the preimage of a maximal compact subgroup of $H^{\text {ad }}(\mathbb{C})$ is a maximal compact subgroup of $\widetilde{H}(\mathbb{C})$, whose volume with respect to the measure determined by $\widetilde{\omega}_{\mathbb{C}}$ is one. The $\omega_{i}$ are, therefore, determined up to multiplication by a non-zero multiplicative constant. We choose this constant so that the volume of any maximal compact subgroup of $H_{i}(\mathbb{C})$ is one. We denote the measures determined on the $H_{i}(\mathbb{R})$ by $\mu_{i}$. Since $K_{\widetilde{H}}^{m}$ is maximal, it is a product of maximal compact open subgroups

$$
K_{H_{i}}^{m} \subset H_{i}\left(\mathbb{A}_{f}\right),
$$

each a product of maximal compact open subgroups $K_{H_{i}, p}^{m} \subset H_{i}\left(\mathbb{Q}_{p}\right)$. Hence,

$$
\widetilde{\mu}\left(\Gamma_{\widetilde{H}}^{m} \backslash \widetilde{H}(\mathbb{R})\right)=\prod_{i=1}^{s} \mu_{i}\left(\Gamma_{H_{i}}^{m} \backslash H_{i}(\mathbb{R})\right),
$$

where $\Gamma_{H_{i}}^{m}:=H_{i}(\mathbb{Q}) \cap K_{H_{i}}^{m}$.

By [21, Section 3.3], each $H_{i}$ is of the form $\operatorname{Res}_{K_{i} / \mathbb{Q}} H_{i}^{\prime}$, where $K_{i}$ is a totally real number field and $H_{i}^{\prime}$ is a simply connected, absolutely quasi-simple group. Now,

$$
H_{i}\left(\mathbb{Q}_{p}\right)=\operatorname{Res}_{K_{i} / \mathbb{Q}} H_{i}^{\prime}\left(\mathbb{Q}_{p}\right)=H_{i}^{\prime}\left(K_{i} \otimes_{\mathbb{Q}} \mathbb{Q}_{p}\right)=\prod_{v \mid p} H_{i}^{\prime}\left(K_{i, v}\right),
$$

where the product runs over the places $v$ of $K_{i}$ lying above $p$ and $K_{i, v}$ is the completion of $K_{i}$ with respect to the valuation determined by $v$. Thus, $K_{H_{i}, p}^{m}$ is a product of maximal compact open subgroups $K_{H_{i}^{\prime}, v}^{m} \subset H_{i}^{\prime}\left(K_{i, v}\right)$. 
Since $H_{i}(\mathbb{R})=\prod_{v \mid \infty} H_{i}^{\prime}\left(K_{i, v}\right)$, we can write

$$
\omega_{i}=\wedge_{v \mid \infty} \omega_{i, v}
$$

where $\omega_{i, v}$ is a real, non-zero, left-invariant differential form of maximal degree on $H_{i}^{\prime}\left(K_{i, v}\right)$. We choose the $\omega_{i, v}$ so that the volume of any maximal compact subgroup of $H_{i}^{\prime}(\mathbb{C})$ is one. Note that, by [16, Section 3.5], for each archimedean place $v$ of $K_{i}$, the Haar measure $\mu_{i, v}$ determined by $\omega_{i, v}$ on $H_{i}^{\prime}(\mathbb{R})$ is precisely that defined in [16, Section 3.6]. Therefore, by [16, Theorem 3.7],

$\mu_{i}\left(\Gamma_{H_{i}}^{m} \backslash H_{i}(\mathbb{R})\right)=D_{K_{i}}^{\frac{1}{2} \operatorname{dim} H_{i}^{\prime}} \cdot\left|N_{K_{i} / \mathbb{Q}}\left(\Delta_{L_{i} / K_{i}}\right)\right|^{\frac{s_{i}}{2}} \cdot\left(\prod_{j=1}^{r_{i}} \frac{m_{i, j} !}{(2 \pi)^{m_{i, j}+1}}\right)^{\left[K_{i}: \mathbb{Q}\right]} \cdot \tau_{K_{i}}\left(H_{i}^{\prime}\right) \cdot \xi_{i}$,

where

- $D_{K_{i}}$ is the absolute value of $\operatorname{disc}\left(K_{i}\right)$,

- $L_{i}$ is the splitting field of the quasi-split inner form $\mathscr{H}_{i}$ of $H_{i}^{\prime}$,

- $N_{K_{i} / \mathbb{Q}}$ is the norm on $K_{i}$,

- $\Delta_{L_{i} / K_{i}}$ is the relative discriminant of $L_{i}$ over $K_{i}$,

- $s_{i}$ is the integer defined in [16, Section 0.4],

- $r_{i}$ is the absolute rank of $\mathscr{H}_{i}$,

- the $m_{i, j}$ are the exponents of the simple, simply connected, compact real-analytic Lie group of the same type as $\mathscr{H}_{i}$,

- $\tau_{K_{i}}\left(H_{i}^{\prime}\right)=1$ is the Tamagawa number of $H_{i}^{\prime}$ (see [16, Section 3.3]),

- $\xi_{i}$ is the product, over all finite places $v$ of $K_{i}$, of local factors $\xi_{i, v}$.

Note first that $\operatorname{dim} H_{i}^{\prime}, s_{i}, r_{i}$, the $m_{i, j}$ and $\left[K_{i}: \mathbb{Q}\right]$ are all positive integers, with the possible exception of $s_{i}$ when $L_{i}=K_{i}$, in which case it becomes irrelevant. It is also worth noting that they are all uniformly bounded.

Recall that $\Delta_{L_{i} / K_{i}}$ is an ideal in $\mathcal{O}_{K_{i}}$ with the property that the prime ideals dividing it are precisely those that ramify in $\mathcal{O}_{L_{i}}$, i.e. those places $v$ of $K_{i}$ such that $\mathscr{H}_{i, K_{i, v}}$ does not split over an unramified extension of $K_{i, v}$. Its norm $N_{K_{i} / \mathbb{Q}}\left(\Delta_{L_{i} / K_{i}}\right)$ is divisible by precisely those primes $p$ such that there exists a place $v$ lying above $p$ and dividing $\Delta_{L_{i} / K_{i}}$.

By [16, Section 2.10], $\xi_{i, v}>1$ for all non-archimedean places $v$ of $K_{i}$. Furthermore, if

- $H_{i, K_{i, v}}^{\prime}$ is not quasi-split,

- $K_{H_{i}^{\prime}, v}^{m}$ is not special,

or

- $H_{i, K_{i, v}}^{\prime}$ splits over an unramified extension of $K_{i, v}$ and $K_{H_{i}^{\prime}, v}^{m}$ is not hyperspecial,

then

$$
\xi_{i, v} \geq q_{i, v}^{r_{i, v}+1} \cdot\left(q_{i, v}+1\right)^{-1}
$$

where $q_{i, v}$ is the cardinality of the residue field $k_{i, v}$ of $K_{i, v}$ and $r_{i, v} \geq 1$ is the rank of $\mathscr{H}_{i, K_{i, v}}$ over the maximal unramified extension of $K_{i, v}$. Therefore, let $\Sigma_{i}$ be the set of primes $p$ such 
that, for some place $v$ of $K_{i}$ lying above $p$, either of the following holds:

- $H_{i, K_{i, v}}^{\prime}$ is not quasi-split,

- $H_{i, K_{i, v}}^{\prime}$ does not split over an unramified extension of $K_{i, v}$,

- $K_{H_{i}^{\prime}, v}^{m}$ is not a hyperspecial subgroup of $H_{i}^{\prime}\left(K_{i, v}\right)$.

Then there exist uniform constants $c_{6}$ and $\delta_{2}$ such that

$$
\mu_{i}\left(\Gamma_{H_{i}}^{m} \backslash H_{i}(\mathbb{R})\right)>c_{6} D_{K_{i}}^{\frac{1}{2} \operatorname{dim} H_{i}^{\prime}} \cdot \prod_{p \in \Sigma_{i}} p^{\delta_{2}} .
$$

However, recall that

$$
H_{i, \mathbb{Q}_{p}}=\prod_{v \mid p} \operatorname{Res}_{K_{i, v} / \mathbb{Q}_{p}} H_{i, K_{i, v}}^{\prime}
$$

Therefore, by [1, Section 6.19], the set $\Sigma\left(H_{i}\right)$ is contained in the union of $\Sigma_{i}$ and the set of primes $p$ dividing $D_{K_{i}}$. On the other hand, suppose that $K_{H_{i}^{\prime}, v}^{m}$ is a hyperspecial subgroup of $H_{i}^{\prime}\left(K_{i, v}\right)$ for each place $v$ of $K_{i}$ lying above a prime $p$.

For each such subgroup, there exists a smooth group scheme $\mathbf{H}_{i, \mathcal{O}_{K_{i, v}}^{\prime}}$ over $\mathcal{O}_{K_{i, v}}$ with generic fibre $H_{i, K_{i, v}}^{\prime}$ such that

- $\mathbf{H}_{i, k_{i, v}}^{\prime}$ is reductive,

- $\mathbf{H}_{i, \mathcal{O}_{k_{i, v}}^{\prime}}^{\prime}\left(\mathcal{O}_{K_{i, v}}\right)$ is equal to $K_{H_{i}^{\prime}, v}^{m}$.

Let

$$
\mathbf{H}_{i, \mathbb{Z}_{p}}:=\prod_{v \mid p} \operatorname{Res}_{\mathcal{O}_{K_{i, v}} / \mathbb{Z}_{p}} \mathbf{H}_{i, \mathcal{O}_{K_{i, v}}^{\prime}}
$$

Then, the generic fibre of $\mathbf{H}_{i, \mathbb{Z}_{p}}$ is $H_{i, \mathbb{Q}_{p}}$ and $\mathbf{H}_{i, \mathbb{Z}_{p}}\left(\mathbb{Z}_{p}\right)=K_{H_{i}, p}^{m}$. Furthermore, if $p$ does not divide $D_{K_{i}}$, then

$$
\mathbf{H}_{i, \mathbb{F}_{p}}=\prod_{v \mid p} \operatorname{Res}_{\mathcal{O}_{K_{i, v}} \otimes_{\mathbb{Z}_{p}} \mathbb{F}_{p} / \mathbb{F}_{p}} \mathbf{H}_{i, \mathcal{O}_{K_{i, v}} \otimes_{\mathbb{Z}_{p}} \mathbb{F}_{p}}=\prod_{v \mid p} \operatorname{Res}_{k_{i, v} / \mathbb{F}_{p}} \mathbf{H}_{i, k_{i, v}}^{\prime}
$$

is a reductive group over $\mathbb{F}_{p}$. Therefore, the set $\Sigma\left(K_{H_{i}}^{m}\right)$ is also contained in the union of the $\Sigma_{i}$ and the set of primes $p$ dividing $D_{K_{i}}$, from which we conclude there exists a uniform constant $\delta_{3}$ such that

$$
\mu_{i}\left(\Gamma_{H_{i}}^{m} \backslash H_{i}(\mathbb{R})\right)>c_{6} \cdot \Pi\left(H_{i}, K_{H_{i}}^{m}\right)^{\delta_{3}} .
$$

However, the union of the $\Sigma\left(H_{i}\right)$ is equal to $\Sigma(H)$ and the union of the $\Sigma\left(K_{H_{i}}^{m}\right)$ is equal to $\Sigma\left(K_{\widetilde{H}}^{m}\right)$.

We will require the following lemma in the proof of Lemma 6.6 and also to obtain suitable Hecke correspondences:

Lemma 6.5. Let $T$ be a maximal torus of $H_{\mathbb{Q}_{p}}$. There exists a basis of $X^{*}(T)$ such that the coordinates of the characters of $T$ intervening in $\overline{\mathbb{Q}}_{p}^{n}$ are bounded in absolute value by a uniform constant. 
Proof. By the proof of [3, Proposition 2.1], since $H$ is the generic Mumford-Tate group on $X_{H}$, there exists a dense set of special points $X_{H}^{\prime}$ in $X_{H}$ such that, for $x \in X_{H}^{\prime}$, the Mumford-Tate group MT(x) of $x$ is a maximal torus in $H$. Choose an $x \in X_{H}^{\prime}$ and let $M:=\operatorname{MT}(x)$. Denote by $L$ the splitting field of $M$ and by $R_{L}$ the torus $\operatorname{Res}_{L / \mathbb{Q}} \mathbb{G}_{m, L}$.

The reciprocity morphism $r_{x}: R_{L} \rightarrow M$ corresponding to $x$ (see [11, p. 104]) is surjective and induces an embedding

$$
X^{*}(M) \hookrightarrow X^{*}\left(R_{L}\right) .
$$

Enumerate the elements $\sigma \in \operatorname{Gal}(L / \mathbb{Q})$, thereby producing a basis $\mathcal{B}:=\left\{b_{\sigma}\right\}$ of $X^{*}\left(R_{L}\right)$. By [23, Section 2], with respect to this basis, the characters of $M$ intervening in $\overline{\mathbb{Q}}^{n}$ have coordinates bounded in absolute value by a uniform constant.

Since any two maximal tori of $H_{\overline{\mathbb{Q}}_{p}}$ are conjugate by an element of $H\left(\overline{\mathbb{Q}}_{p}\right)$, we may conjugate $r_{x, \overline{\mathbb{Q}}_{p}}$ by an element of $H\left(\overline{\mathbb{Q}}_{p}\right)$ to obtain a surjective morphism

$$
r_{x, \overline{\mathbb{Q}}_{p}}^{\prime}: R_{L, \overline{\mathbb{Q}}_{p}} \rightarrow T_{\overline{\mathbb{Q}}_{p}}
$$

Thus, we obtain an embedding

$$
X^{*}\left(T_{\overline{\mathbb{Q}}_{p}}\right) \hookrightarrow X^{*}\left(R_{L, \overline{\mathbb{Q}}_{p}}\right)
$$

such that, with respect to the basis $\mathscr{B}$, the coordinates of the characters of $T$ intervening in $\overline{\mathbb{Q}}_{p}^{n}$ are uniformly bounded in absolute value. Since our representation was faithful, these characters generate $X^{*}\left(T_{\mathbb{Q}_{p}}\right)$ and so there are only finitely many possibilities for this submodule of $X^{*}\left(R_{L}, \overline{\mathbb{Q}}_{p}\right)$. For each such possibility, choose a basis for $X^{*}\left(T_{\overline{\mathbb{Q}}_{p}}\right)$ and consider the maximum of the absolute values of coordinates of the characters intervening in $\overline{\mathbb{Q}}_{p}^{n}$ with respect to these bases.

Lemma 6.6. There exist uniform constants $c_{7}$ and $c_{8}$ such that, for any $p \notin \Sigma\left(\widetilde{H}, K_{\widetilde{H}}^{m}\right)$ greater than $c_{7}$, such that $\widetilde{K}_{H, p} \subsetneq K_{\widetilde{H}, p}^{m}$,

$$
\left[K_{\widetilde{H}, p}^{m}: \widetilde{K}_{H, p}\right]>c_{8} p .
$$

Proof. We will imitate the proof of [19, Proposition 3.15]. Let $p \notin \Sigma\left(\widetilde{H}, K_{\widetilde{H}}^{m}\right)$ be a prime. Since

$$
K_{\widetilde{H}, p}^{m} \subset \widetilde{H}\left(\mathbb{Q}_{p}\right)
$$

is hyperspecial and $H_{\mathbb{Q}_{p}}$ is unramified, there exist smooth reductive group schemes $\widetilde{\mathbf{H}}$ and $\mathbf{H}$ over $\mathbb{Z}_{p}$, the generic fibres of which are $\widetilde{H}_{\mathbb{Q}_{p}}$ and $H_{\mathbb{Q}_{p}}$, such that

$$
K_{\widetilde{H}, p}^{m}=\widetilde{\mathbf{H}}\left(\mathbb{Z}_{p}\right) \text {. }
$$

By [20, Lemma 2.3.1], the central isogeny $\pi_{\mathbb{Q}_{p}}$ extends uniquely to a central isogeny

$$
\pi_{\mathbb{Z}_{p}}: \widetilde{\mathbf{H}} \rightarrow \mathbf{H}
$$

and we denote the kernel $\mathbf{Z}$.

The map

$$
\widetilde{K}_{H, p} \backslash \widetilde{\mathbf{H}}\left(\mathbb{Z}_{p}\right) \rightarrow K_{H, p} \backslash \mathbf{H}\left(\mathbb{Z}_{p}\right)
$$

is injective. However, recall from the proof of Lemma 6.3 that the cokernel is no larger than $H^{1}\left(\operatorname{Gal}\left(\mathbb{Q}_{p}^{\text {un }} / \mathbb{Q}_{p}\right), \mathbf{Z}\left(\mathbb{Z}_{p}^{\text {un }}\right)\right)$. Furthermore, if $F$ is the splitting field of $Z$ and $v$ is a place of $F$ lying above $p$, the kernel of the restriction map to $H^{1}\left(\operatorname{Gal}\left(\mathbb{Q}_{p}^{\text {un }} / F_{v}\right), \mathbf{Z}\left(\mathbb{Z}_{p}^{\text {un }}\right)\right)$ is uniformly 
bounded. However, as we have seen, $H^{1}\left(\operatorname{Gal}\left(\mathbb{Q}_{p}^{\text {un }} / F_{v}\right), \mathbf{Z}\left(\mathbb{Z}_{p}^{\text {un }}\right)\right)$ is itself uniformly bounded. Therefore, it suffices to show there exist uniform constants $c_{7}$ and $c_{8}$ such that

$$
\left[\mathbf{H}\left(\mathbb{Z}_{p}\right): K_{H, p}\right]>c_{8} p
$$

whenever $p>c_{7}$.

Let $\mathbf{T}$ be a maximal torus of $\mathbf{H}$. The group $\left(K_{H, p} \cap \mathbf{T}\left(\mathbb{Z}_{p}\right)\right) \backslash \mathbf{T}\left(\mathbb{Z}_{p}\right)$ is a subset of $K_{H, p} \backslash \mathbf{H}\left(\mathbb{Z}_{p}\right)$ and so the previous lower bound for the size of this group would suffice. Let $T$ denote the generic fibre of $\mathbf{T}$ and note that, by [18, Section 3.8.2], the hyperspecial subgroup $\mathbf{T}\left(\mathbb{Z}_{p}\right)$ is the maximal compact subgroup of $T\left(\mathbb{Q}_{p}\right)$. Therefore, if $K_{p}=G_{\mathbb{Z}_{p}}\left(\mathbb{Z}_{p}\right)$, a condition satisfied for all primes $p$ greater than a uniform constant, $T_{\mathbb{Z}_{p}}$ is only a torus if

$$
K_{T, p}:=\mathrm{GL}_{n}\left(\mathbb{Z}_{p}\right) \cap T\left(\mathbb{Q}_{p}\right)=K_{H, p} \cap T\left(\mathbb{Q}_{p}\right)=K_{H, p} \cap \mathbf{T}\left(\mathbb{Z}_{p}\right)
$$

is equal to $\mathbf{T}\left(\mathbb{Z}_{p}\right)$.

We claim that it is possible to choose $\mathbf{T}$ such that $T_{\mathbb{Z}_{p}}$ is not a torus. In particular, since, by [7, Exposé XXII, Section 8], every semisimple element of $\mathbf{H}$ is contained in a maximal torus, we are claiming that $\mathbf{H}\left(\mathbb{Z}_{p}\right) \backslash K_{H, p}$ contains a semisimple element. To see this, note that, by [7, Exposé XXII, Corollaire 1.10], the functor of maximal tori of $\mathbf{H}$ is representable by $\mathbf{H} / \mathbf{N}$, where $\mathbf{N}$ is the normaliser of a maximal torus in $\mathbf{H}$. By [7, Exposé XXII, paragraph following Lemme 4.5], and by [7, Exposé XXI, Proposition 5.9], the universal maximal torus $\mathbf{T}$ of $\mathbf{H}$ (see [7, Exposé XXII, Section 8]) has the same dimension as H. However, the morphism $u: \underline{\mathbf{T}} \rightarrow \mathbf{H}$ is quasi-finite. Hence, by [7, Exposé XXII, Proposition 8.1], the semisimple elements of $\mathbf{H}$ constitute a constructible set of dimension $\operatorname{dim} \mathbf{H}$, which therefore contains a Zariski open set. On the other hand, $\mathbf{H}\left(\mathbb{Z}_{p}\right) \backslash K_{H, p}$ is open in $\mathbf{H}\left(\mathbb{Z}_{p}\right)$ for the $p$-adic topology and so the claim follows.

By [15, Section 3.3, p. 134], every maximal compact subgroup of $\mathrm{GL}_{n}\left(\mathbb{Q}_{p}\right)$ is conjugate to $\mathrm{GL}_{n}\left(\mathbb{Z}_{p}\right)$ by an element of $\mathrm{GL}_{n}\left(\mathbb{Q}_{p}\right)$. Hence, there exists a $g \in \mathrm{GL}_{n}\left(\mathbb{Q}_{p}\right)$ such that

$$
\mathbf{T}\left(\mathbb{Z}_{p}\right)=g \mathrm{GL}_{n}\left(\mathbb{Z}_{p}\right) g^{-1} \cap T\left(\mathbb{Q}_{p}\right) .
$$

Let $T_{0}:=g^{-1} T g$. Hence, $\mathrm{GL}_{n}\left(\mathbb{Z}_{p}\right) \cap T_{0}\left(\mathbb{Q}_{p}\right)$ is a maximal compact open subgroup $K_{T_{0}, p}^{m}$ of $T_{0}\left(\mathbb{Q}_{p}\right)$ and, since $K_{T, p}=\mathrm{GL}_{n}\left(\mathbb{Z}_{p}\right) \cap T\left(\mathbb{Q}_{p}\right)$, conjugation by $g^{-1}$ establishes a bijection

$$
K_{T, p} \backslash \mathbf{T}\left(\mathbb{Z}_{p}\right) \leftrightarrow\left(g^{-1} \mathrm{GL}_{n}\left(\mathbb{Z}_{p}\right) g \cap T_{0}\left(\mathbb{Q}_{p}\right)\right) \backslash K_{T_{0}, p}^{m} .
$$

The latter index is the size of the orbit $K_{T_{0}, p}^{m} \cdot g^{-1} \mathbb{Z}_{p}^{n}$ in the space of lattices of $\mathbb{Q}_{p}^{n}$. Note that

$$
K_{T_{0}, p}^{m}=T_{0, \mathbb{Z}_{p}}\left(\mathbb{Z}_{p}\right)
$$

Since $T$ splits over an unramified extension of $\mathbb{Q}_{p}$, so too does $T_{0}$ and so, by [18, Section 3.8.2], $K_{T_{0}, p}^{m}$ is a hyperspecial subgroup. Therefore, $T_{0, \mathbb{Z}_{p}}$ is a torus.

By [7, Exposé X, Lemme 4.1], there is a canonical isomorphism

$$
X^{*}\left(T_{0, \overline{\mathbb{Q}}_{p}}\right) \cong X^{*}\left(T_{0, \overline{\mathbb{F}}_{p}}\right)
$$

identifying the characters intervening in $\overline{\mathbb{Q}}_{p}^{n}$ and $\overline{\mathbb{F}}_{p}^{n}$. Thus, with respect to the image of the basis obtained using Lemma 6.5 , the coordinates of the characters of $T_{0, \mathbb{F}_{p}}$ intervening in $\overline{\mathbb{F}}_{p}^{n}$ are bounded in absolute value by a uniform constant. 
Therefore, by [8, Lemma 4.4.1], for all subspaces $W$ of $\overline{\mathbb{F}}_{p}^{n}$, the group of connected components of the stabiliser of $W$ in $T_{0, \overline{\mathbb{F}}_{p}}$ is of order bounded by a uniform constant. Since $T_{\mathbb{Z}_{p}}$ is not a torus, $T_{0, \mathbb{Z}_{p}}$ does not fix the lattice $g^{-1} \mathbb{Z}_{p}^{n}$ in the sense of [8, Section 3.3]. Therefore, [8, Proposition 4.3.9] implies that there exists a uniform constant $c_{8}$ such that the size of the orbit $T_{0, \mathbb{Z}_{p}}\left(\mathbb{Z}_{p}\right) \cdot g^{-1} \mathbb{Z}_{p}^{n}$ is greater than $c_{8} p$.

Lemma 6.7. There exists a uniform constant $c_{9}$ such that, if $p \notin \Sigma\left(\widetilde{H}, \widetilde{K}_{H}\right)$ is a prime greater than $c_{9}$, then $p \notin \Sigma\left(H, K_{H}\right)$.

Proof. Since $H_{\mathbb{Q}_{p}}$ is unramified and $\widetilde{K}_{H, p}$ is hyperspecial, there exist smooth reductive group schemes $\widetilde{\mathbf{H}}$ and $\mathbf{H}$ over $\mathbb{Z}_{p}$, the generic fibres of which are $\widetilde{H}_{\mathbb{Q}_{p}}$ and $H_{\mathbb{Q}_{p}}$, such that

$$
\widetilde{K}_{H, p}=\widetilde{\mathbf{H}}\left(\mathbb{Z}_{p}\right) .
$$

Therefore, $\mathbf{H}\left(\mathbb{Z}_{p}\right)$ is a hyperspecial subgroup of $H\left(\mathbb{Q}_{p}\right)$. By [20, Lemma 2.3.1], the central isogeny $\pi_{\mathbb{Q}_{p}}$ extends uniquely to a central isogeny $\pi_{\mathbb{Z}_{p}}: \widetilde{\mathbf{H}} \rightarrow \mathbf{H}$.

Let $K_{H, p}^{m}$ be a maximal compact open subgroup containing $K_{H, p}$. Therefore, $K_{H, p}^{m}$ contains the image of $\widetilde{K}_{H, p}$. Since, by [18, Section 3.8.2], $\widetilde{K}_{H, p}$ is maximal, [14, Proposition 3.3] implies that

$$
K_{H, p}^{m}=\mathbf{H}\left(\mathbb{Z}_{p}\right) .
$$

Since $\widetilde{K}_{H, p}=\widetilde{\mathbf{H}}\left(\mathbb{Z}_{p}\right)$, the map

$$
\widetilde{\mathbf{H}}\left(\mathbb{Z}_{p}\right) \rightarrow K_{H, p} \backslash \mathbf{H}\left(\mathbb{Z}_{p}\right)
$$

is trivial and we have seen that the cokernel is uniformly bounded. On the other hand, the proof of Lemma 6.6 shows that, if $p$ is greater than a uniform constant and $K_{H, p} \subsetneq \mathbf{H}\left(\mathbb{Z}_{p}\right)$, then $\left[\mathbf{H}\left(\mathbb{Z}_{p}\right): K_{H, p}\right]$ is at least a uniform constant times $p$.

Proof of Theorem 6.1. Recall that

$$
\mu\left(\Gamma_{H} \backslash X_{H}^{+}\right)=\frac{\left[\operatorname{ad} \circ \pi\left(\Gamma_{\widetilde{H}}^{m}\right): \operatorname{ad} \circ \pi\left(\widetilde{\Gamma}_{H}\right)\right]}{\left[\operatorname{ad}\left(\Gamma_{H}\right): \operatorname{ad} \circ \pi\left(\widetilde{\Gamma}_{H}\right)\right]} \cdot \mu\left(\operatorname{ad} \circ \pi\left(\Gamma_{\widetilde{H}}^{m}\right) \backslash H^{\operatorname{ad}}(\mathbb{R})^{+}\right) .
$$

Therefore, by Lemmas 6.2 and 6.3, we have

$$
\mu\left(\Gamma_{H} \backslash X_{H}^{+}\right)>c_{3} c_{4}^{-1} C^{-\left|\Sigma\left(H, K_{H}\right)\right|} \cdot\left[K_{\widetilde{H}}^{m}: \widetilde{K}_{H}\right] \cdot \mu\left(\operatorname{ad} \circ \pi\left(\Gamma_{\widetilde{H}}^{m}\right) \backslash H^{\mathrm{ad}}(\mathbb{R})^{+}\right),
$$

so, by Lemma 6.4,

$$
\mu\left(\Gamma \backslash X_{H}^{+}\right)>c_{3} c_{4}^{-1} c_{5} C^{-\left|\Sigma\left(H, K_{H}\right)\right|} \cdot\left[K_{\widetilde{H}}^{m}: \widetilde{K}_{H}\right] \cdot \Pi\left(\widetilde{H}, K_{\widetilde{H}}^{m}\right)^{\delta_{1}} .
$$

Lemma 6.6 implies that there exist uniform constants $c_{10}$ and $\delta_{4}$ such that

$$
\mu\left(\Gamma \backslash X_{H}^{+}\right)>c_{10} C^{-\left|\Sigma\left(H, K_{H}\right)\right|} \cdot \Pi\left(\widetilde{H}, \widetilde{K}_{H}\right)^{\delta_{4}} .
$$

Therefore, the result follows from Lemma 6.7.

Proof of Theorem 1.4. Follows from Theorem 5.1 and Theorem 6.1. 


\section{Choosing a suitable Hecke correspondence}

In this section, we prove an analogue of [9, Theorem 8.1], demonstrating the existence of suitable Hecke correspondences. Recall that, if $(G, X)$ is a Shimura datum, $G^{\text {ad }}$ decomposes into a product of simple factors, which we denote $G_{i}$. Thus, $X_{\text {ad }}$ decomposes into a product of factors $X_{i}$ and, if $X^{+}$is a connected component of $X$, then it decomposes into a product of factors $X_{i}^{+}$. If $K_{\mathrm{ad}}$ is equal to a product of compact open subgroups $K_{i} \subset G_{i}\left(\mathbb{A}_{f}\right)$, then $S_{K_{\text {ad }}}\left(G^{\text {ad }}, X_{\text {ad }}\right)$ is equal to the product of the $S_{K_{i}}\left(G_{i}, X_{i}\right)$. If $K$ is a compact open subgroup of $G\left(\mathbb{A}_{f}\right)$, equal to the product of compact open subgroups $K_{l}$ of $G\left(\mathbb{Q}_{l}\right)$, we will use the notation $K^{p}$ to denote the product $\prod_{l \neq p} K_{l}$.

Theorem 7.1. Let $\left(G^{\prime}, X^{\prime}\right)$ be a Shimura datum such that $G^{\prime}=G^{\prime a d}$, let $K^{\prime}$ be a neat compact open subgroup of $G^{\prime}\left(\mathbb{A}_{f}\right)$, equal to a product of compact open subgroups $K_{p}^{\prime}$ of $G^{\prime}\left(\mathbb{Q}_{p}\right)$, and fix a faithful representation

$$
\rho: G^{\prime} \hookrightarrow \mathrm{GL}_{n}
$$

There exist positive integers $k$ and $f$ such that, if

- $V$ is a strongly special subvariety of $S_{K^{\prime}}\left(G^{\prime}, X^{\prime}\right)$, defined by $\left(H, X_{H}\right)$,

- $p \notin \Sigma\left(H, K_{H}\right)$ is a prime such that $K_{p}^{\prime}=G_{\mathbb{Z}_{p}}^{\prime}\left(\mathbb{Z}_{p}\right)$,

- $(G, X)$ is a Shimura subdatum of $\left(G^{\prime}, X^{\prime}\right)$ such that $V$ is contained in $S_{K}(G, X)$, where $K:=K^{\prime} \cap G\left(\mathbb{A}_{f}\right)$,

then there exist a compact open subgroup

$$
I_{p} \subset K_{p}:=K_{p}^{\prime} \cap G\left(\mathbb{Q}_{p}\right)
$$

and an element $\alpha \in G\left(\mathbb{Q}_{p}\right)$ such that

- $\left[K_{p}: I_{p}\right] \leq p^{f}$,

- if $I \subset K$ is the compact open subgroup $K^{p} I_{p} \subset G\left(\mathbb{A}_{f}\right)$,

$$
\tau: \operatorname{Sh}_{I}(G, X) \rightarrow \operatorname{Sh}_{K}(G, X)
$$

is the natural morphism, and $\widetilde{V} \subset S_{I}(G, X)_{\mathbb{C}}$ is an irreducible component of $\tau^{-1}(V)$, then $\widetilde{V} \subset T_{\alpha}(\widetilde{V})$,

- for every $k_{1}, k_{2} \in I_{p}$, the image of $k_{1} \alpha k_{2}$ generates an unbounded subgroup of $G_{i}\left(\mathbb{Q}_{p}\right)$ for each $i$,

- $\left[I_{p}: I_{p} \cap \alpha I_{p} \alpha^{-1}\right]<p^{k}$.

In this section, we will use the term uniform to mean depending only on $\left(G^{\prime}, X^{\prime}\right), K^{\prime}$ and $\rho$. Firstly, we will deal with the matter of including a strongly special subvariety in its image under a Hecke correspondence:

Lemma 7.2. There exists a uniform integer $A$ such that, for any $\alpha \in H\left(\mathbb{A}_{f}\right)$,

$$
V \subset T_{\alpha^{A}}(V)
$$


Proof. By definition, the subvariety $V$ is the image of $X_{H}^{+} \times\{1\}$ in $\operatorname{Sh}_{K}(G, X)$. Thus, consider a point $\overline{(x, 1)} \in V$ with $x \in X_{H}^{+}$. Let

$$
\pi: \widetilde{H} \rightarrow H
$$

be the simply connected covering, whose degree we denote $d$, and consider an $\alpha \in H\left(\mathbb{A}_{f}\right)$. Therefore, for any positive integer $A$ divisible by $d$, there exists an element $\beta \in \widetilde{H}\left(\mathbb{A}_{f}\right)$ such that $\pi(\beta)=\alpha^{A}$. By strong approximation applied to $\widetilde{H}, \beta=q k$, where $q \in \widetilde{H}(\mathbb{Q})$ and $k \in \pi^{-1}(K)$. Note that, since $\pi$ is proper, $\pi^{-1}(K)$ is a compact open subgroup of $\widetilde{H}\left(\mathbb{A}_{f}\right)$. Since $\widetilde{H}(\mathbb{R})$ is connected, $\pi(q) \in H(\mathbb{R})^{+}$and $\pi(q) \cdot x \in X_{H}^{+}$.

Thus, consider the point

$$
\overline{(\pi(q) \cdot x, \pi(\beta))} \in T_{\alpha^{A}}(V) .
$$

By the previous discussion, this is equal to $\overline{(x, 1)}$. Since $d$ is bounded by a uniform integer $D$, setting $A=D$ ! finishes the proof.

In order to find suitable Hecke correspondences, we will also need the following two results on maximal split tori:

Lemma 7.3. Let $p \notin \Sigma\left(H, K_{H}\right)$ be a prime such that $K_{p}^{\prime}=G_{\mathbb{Z}_{p}}^{\prime}\left(\mathbb{Z}_{p}\right)$. Then there exists a maximal split torus $S \subset H_{\mathbb{Q}_{p}}$ such that $S_{\mathbb{Z}_{p}}$ is a torus.

Proof. Since $p \notin \Sigma\left(H, K_{H}\right)$, there exists a smooth reductive group scheme $\mathbf{H}$ over $\mathbb{Z}_{p}$ such that $\mathbf{H}_{\mathbb{Q}_{p}}=H_{\mathbb{Q}_{p}}$ and $\mathbf{H}\left(\mathbb{Z}_{p}\right)=K_{H, p}$. Let $\mathbf{S}$ be a maximal split torus of $\mathbf{H}$ and let $S$ denote its generic fibre. By [18, Section 3.8.1], $S_{\mathbb{Z}_{p}}$ is a torus if

$$
S_{\mathbb{Z}_{p}}\left(\mathbb{Z}_{p}\right):=\operatorname{GL}_{n}\left(\mathbb{Z}_{p}\right) \cap S\left(\mathbb{Q}_{p}\right)=K_{H, p} \cap S\left(\mathbb{Q}_{p}\right)
$$

is equal to $\mathbf{S}\left(\mathbb{Z}_{p}\right)$. However, since $K_{H, p}=\mathbf{H}\left(\mathbb{Z}_{p}\right)$, it follows that $S_{\mathbb{Z}_{p}}\left(\mathbb{Z}_{p}\right)$ contains $\mathbf{S}\left(\mathbb{Z}_{p}\right)$ and so, by [18, Section 3.8.2], they are equal.

Lemma 7.4. Assume $H_{\mathbb{Q}_{p}}$ is quasi-split and let $S \subset H_{\mathbb{Q}_{p}}$ be a maximal split torus. There exists a basis of $X^{*}(S)$ such that the coordinates of the characters of $S$ that intervene in $\mathbb{Q}_{p}^{n}$ are uniformly bounded in absolute value.

Proof. Let $T \subset H_{\mathbb{Q}_{p}}$ be the centraliser of $S$ in $H_{\mathbb{Q}_{p}}$. Since $H_{\mathbb{Q}_{p}}$ is quasi-split, $T$ is a maximal torus of $H_{\mathbb{Q}_{p}}$. By [22, Section 7.4], there exists an isogeny $T \rightarrow S \times A$, where $A$ is the maximal anisotropic subtorus of $T$, and the degree $d$ of this isogeny is bounded by $\left[L_{T}: \mathbb{Q}\right]^{\operatorname{dim} T}$, where $L_{T}$ is the splitting field of $T$. Note that $\operatorname{dim} T$ is bounded by the absolute rank of $G$ and that, by $\left[6\right.$, Theorem 5.1], $\left[L_{T}: \mathbb{Q}\right]$ is bounded in terms of the dimension of $T$.

Consider the map of characters

$$
\varphi: \chi \mapsto \chi_{S}+\chi_{A}: X^{*}(T) \rightarrow X^{*}(S) \oplus X^{*}(A)
$$

induced by the inclusions $S \subset T$ and $A \subset T$. The characters of $S$ intervening in $\mathbb{Q}_{p}^{n}$ are precisely the $\chi_{S}$ such that $\chi \in X^{*}(T)$ intervenes in $\overline{\mathbb{Q}}_{p}^{n}$.

Now consider the embedding

$$
\phi: X^{*}(S) \oplus X^{*}(A) \hookrightarrow X^{*}(T)
$$


induced by the above isogeny. By Lemma 6.5 there exists a basis $\left\{e_{1}, \ldots, e_{r}\right\}$ of $X^{*}(T)$ such that the coordinates of the characters of $T$ intervening in $\overline{\mathbb{Q}}_{p}^{n}$ are bounded in absolute value by a uniform constant $B^{\prime}$. Given a character of $T$, its coordinates increase in absolute value by at most a factor of $d$ under $\phi \circ \varphi$.

Thus, let $\left\{\chi_{i}\right\}$ be the characters of $T$ intervening in $\overline{\mathbb{Q}}_{p}^{n}$ and let $\left\{\chi_{i, S}+\chi_{i, A}\right\}$ be their images in $X^{*}(S) \oplus X^{*}(A)$. Write the image of $\chi_{i, S}+\chi_{i, A}$ under $\phi$ as

$$
\sum_{j=1}^{r} n_{i, j} e_{j}
$$

Hence, $\left|n_{i, j}\right|<B:=d B^{\prime}$ for all $i$ and $j$ and $n_{i, j}=n_{i, S, j}+n_{i, A, j}$, where

$$
\sum_{j=1}^{r} n_{i, S, j} e_{j} \quad \text { and } \quad \sum_{j=1}^{r} n_{i, A, j} e_{j}
$$

are the images of the $\chi_{i, S}$ and $\chi_{i, A}$ under $\phi$, respectively. Therefore, either $\left|n_{i, S, j}\right|<B$ for all $i$ and $j$, or there exist $i$ and $j$ such that $\left|n_{i, S, j}\right| \geq B$, in which case $n_{i, S, j}$ and $n_{i, A, j}$ are of opposite signs.

Assume the latter, letting $\chi_{i}$ denote the corresponding character and letting $n_{i, S, j}$ denote the coefficient with absolute value at least $B$. Since our representation of $T$ was defined over $\mathbb{Q}_{p}$, for each $\tau \in \operatorname{Gal}\left(\overline{\mathbb{Q}}_{p} / \mathbb{Q}_{p}\right), \tau \chi_{i}$ also intervenes. Since $S$ is split,

$$
\tau \chi_{i, S}=\chi_{i, S} \quad \text { for every } \tau \in \operatorname{Gal}\left(\overline{\mathbb{Q}}_{p} / \mathbb{Q}_{p}\right) .
$$

Therefore, the image of $\tau \chi_{i}$ in $X^{*}(S) \oplus X^{*}(A)$ varies over $\chi_{i, S}+\tau \chi_{i, A}$ and, since $A$ is anisotropic,

$$
\sum_{\tau \in \operatorname{Gal}\left(\overline{\mathbb{Q}}_{p} / \mathbb{Q}_{p}\right)} \tau \chi_{i, A}=0 .
$$

Thus, there exists a $\tau \in \operatorname{Gal}\left(\overline{\mathbb{Q}}_{p} / \mathbb{Q}_{p}\right)$ such that the coefficient of $e_{j}$ corresponding to the image of $\tau \chi_{i, A}$ under $\phi$ is of the opposite sign to $n_{i, A, j}$. But then this coefficient is of the same sign as $n_{i, S, j}$, which implies that the sum of these two coefficients has absolute value greater than or equal to $B$, which is a contradiction.

Therefore, with respect to the basis $\left\{e_{1}, \ldots, e_{r}\right\}$ of $X^{*}(T)$, the coordinates of the characters of $S$ intervening in $\mathbb{Q}_{p}^{n}$ are bounded in absolute value by $B$. Since our representation is faithful, these characters generate $X^{*}(S)$ and so, as a submodule of $X^{*}(T)$, there are only finitely many possibilities for $X^{*}(S)$. For each such possibility, choose a basis and consider the maximum of the absolute values of the coordinates of the characters intervening in $\mathbb{Q}_{p}^{n}$.

Proof of Theorem 7.1. By Lemma 7.3, since $p \notin \Sigma\left(H, K_{H}\right)$, we can find a non-trivial, maximal, split torus $S \subset H_{\mathbb{Q}_{p}}$ such that $S_{\mathbb{Z}_{p}}$ is a torus. Furthermore, by Lemma 7.4, there is a basis of $X^{*}(S)$ such that the coordinates of the characters intervening in $\mathbb{Q}_{p}^{n}$ are uniformly bounded in absolute value. Let $\pi_{i}: G \rightarrow G_{i}$ denote the natural morphisms. By [13, SV3], it follows that $S_{i}:=\pi_{i}(S)$ is a non-trivial, split torus.

As $K_{p}^{\prime}=G_{\mathbb{Z}_{p}}^{\prime}\left(\mathbb{Z}_{p}\right)$, the compact open subgroup $K_{p}:=K_{p}^{\prime} \cap G\left(\mathbb{Q}_{p}\right)$ of $G\left(\mathbb{Q}_{p}\right)$ is equal to $G_{\mathbb{Z}_{p}}\left(\mathbb{Z}_{p}\right)$ and

$$
\left[K_{p}: K_{p} \cap \alpha K_{p} \alpha^{-1}\right]=\left[K_{p}: K_{p} \cap \alpha G_{\mathbb{Z}_{p}}\left(\mathbb{Z}_{p}\right) \alpha^{-1}\right] \quad \text { for any } \alpha \in S\left(\mathbb{Q}_{p}\right) .
$$


By [8, Lemma 7.4.3], for $q_{i}=\pi_{i \mid S}$ and $e=A$ (the positive integer given by Lemma 7.2), there exist a uniform constant $k^{\prime}$ and an element $\alpha \in S\left(\mathbb{Q}_{p}\right)$ such that no $\pi_{i}(\alpha)$ lies in a compact subgroup of $S_{i}\left(\mathbb{Q}_{p}\right)$ and

$$
\left[K_{p}: K_{p} \cap \alpha^{A} G_{\mathbb{Z}_{p}}\left(\mathbb{Z}_{p}\right) \alpha^{-A}\right]<p^{k^{\prime}} .
$$

Next we define $I_{p}$ following [9, Section 8.3.2]. Since $S$ is a split torus and $S_{\mathbb{Z}_{p}}$ is a torus, it follows that $G_{\mathbb{Z}_{p}}\left(\mathbb{Z}_{p}\right)=K_{p}$ is in good position with respect to $S$ (using the terminology of [9, Section 4.1.6]).

Let $f$ be the constant, defined in [9, Lemma 8.1.6(b)], for the group $G^{\prime}$. We claim that there exists an Iwahori subgroup $I_{p}^{1}$ of $G\left(\mathbb{Q}_{p}\right)$ such that

$$
\left[K_{p}: K_{p} \cap I_{p}^{1}\right]<p^{f} .
$$

To see this we let $K_{p}^{1}$ be any maximal compact subgroup of $G\left(\mathbb{Q}_{p}\right)$ containing $K_{p}$. Since $K_{p}$ is in good position with respect to $S$, so too is $K_{p}^{1}$. Thus, by [9, Lemma 8.1.6 (b) (i)], there exists an Iwahori subgroup $I_{p}^{1} \subset K_{p}^{1}$ in good position with respect to $S$ satisfying $\left[K_{p}^{1}: I_{p}^{1}\right]<p^{f}$. Thus,

$$
\left[K_{p}: K_{p} \cap I_{p}^{1}\right]<p^{f} .
$$

Let $S^{\prime}$ be a maximal split torus of $G_{\mathbb{Q}_{p}}$ containing $S$ such that $I_{p}^{1}$ is in good position with respect to $S^{\prime}$. Let $M$ be the centraliser of $S^{\prime}$ in $G_{\mathbb{Q}_{p}}$. Let $\mathscr{B}$ be the (extended) Bruhat-Tits building of $G$ and $\mathcal{A} \subset \mathscr{B}$ the apartment of $\mathscr{B}$ associated to $S^{\prime}$.

The group $M\left(\mathbb{Q}_{p}\right)$ acts on $\mathcal{A}$ as follows: we denote by

$$
\operatorname{ord}_{M}: M\left(\mathbb{Q}_{p}\right) \rightarrow X_{*}(M)_{\mathbb{Q}_{p}}
$$

the homomorphism characterised by

$$
\left\langle\operatorname{ord}_{M}(m), \chi\right\rangle=\operatorname{ord}_{p}(\chi(m)) \text { for all } \chi \in X^{*}(M)_{\mathbb{Q}_{p}},
$$

where $\operatorname{ord}_{p}$ is the normalised additive valuation on $\mathbb{Q}_{p}^{*}$ and $X_{*}(M)_{\mathbb{Q}_{p}}\left(\operatorname{resp} . X^{*}(M)_{\mathbb{Q}_{p}}\right)$ denotes the group of cocharacters (resp. characters) of $M$ defined over $\mathbb{Q}_{p}$. Let $\Lambda \subset X_{*}(M)_{\mathbb{Q}_{p}}$ be the free $\mathbb{Z}$-module $\operatorname{ord}_{M}\left(M\left(\mathbb{Q}_{p}\right)\right)$. Then $M\left(\mathbb{Q}_{p}\right)$ acts on $\mathcal{A}$ via $\Lambda$-translations.

Let $K_{p}^{m}$ be a special compact subgroup containing $I_{p}^{1}$ and let $x \in \mathcal{A}$ be the unique special vertex fixed by $K_{p}^{m}$. Recall the element $\alpha \in S\left(\mathbb{Q}_{p}\right)$ chosen above. The vector $\operatorname{ord}_{M}(\alpha) \in \Lambda$ is non-trivial. Let $C$ be the chamber of $\mathcal{A}$ fixed pointwise by $I_{l}^{1}$ (it contains $x$ in its closure). Consider the chamber $C^{\prime}=C+\operatorname{ord}_{M}(\alpha)$. Let $\mathcal{C} \subset \mathcal{A}$ be the unique Weyl chamber with apex $x$ containing $C^{\prime}$. Finally, let $I_{p}^{2}$ be the Iwahori subgroup of $G\left(\mathbb{Q}_{p}\right)$ fixing the unique chamber of $\mathcal{C}$ containing $x$ in its closure.

Define $I_{p}$ as the intersection $K_{p} \cap I_{p}^{1} \cap I_{p}^{2}$. Since $I_{p}^{2}$ stabilises a chamber in $\mathcal{A}$ it is also in good position with respect to $S^{\prime}$ and, therefore, $S$. Thus, $I_{p}$ is in good position with respect to $S$. It follows from [9, Lemma 8.1.6 (b) (ii)] that

$$
\begin{aligned}
{\left[K_{p}: I_{p}\right] } & =\left[K_{p}: K_{p} \cap I_{p}^{1} \cap I_{p}^{2}\right] \\
& \leq\left[K_{p}^{1}: I_{p}^{1} \cap I_{p}^{2}\right] \\
& <p^{f},
\end{aligned}
$$

which is the first condition of Theorem 7.1. By Lemma 7.2, we have $\widetilde{V} \subset T_{\alpha^{A}}(\widetilde{V})$, which is the second condition of Theorem 7.1. 
Let $S_{i}^{\prime}:=\pi_{i}\left(S^{\prime}\right)$ and denote by $M_{i}:=\pi_{i}(M)$ its centraliser. Let $C_{i}$ be the unique chamber of the Bruhat-Tits building $\mathscr{B}_{i}$ of $G_{i, \mathbb{Q}_{p}}$ fixed by the Iwahori subgroup $\pi_{i}\left(I_{p}^{2}\right)$ and let $x_{i}$ be the vertex in the closure of $C_{i}$ fixed by $\pi_{i}\left(K_{p}^{m}\right)$. Finally, let $\ell_{i}$ be the unique Weyl chamber of the apartment $\mathcal{A}_{i}$ corresponding to $S_{i}^{\prime}$ with apex $x_{i}$ and containing $C_{i}$.

For $M_{i}$ we have a homomorphism

$$
\operatorname{ord}_{M_{i}}: M_{i}\left(\mathbb{Q}_{p}\right) \rightarrow X_{*}\left(M_{i}\right)_{\mathbb{Q}_{p}}
$$

defined analogously to $\operatorname{ord}_{M}$. We denote the image $\operatorname{ord}_{M_{i}}\left(M_{i}\left(\mathbb{Q}_{p}\right)\right.$ by $\Lambda_{i}$. Thus, $M_{i}\left(\mathbb{Q}_{p}\right)$ acts on $\mathcal{A}_{i}$ by $\Lambda_{i}$-translations. We denote by $\Lambda_{i}^{+} \subset \Lambda_{i}$ the positive cone stabilising $\mathscr{C}_{i}$. By virtue of our choice of $I_{p}^{2}$, since $\pi_{i}(\alpha)$ does not lie in a compact subgroup of $S_{i}\left(\mathbb{Q}_{p}\right)$, it follows that $\operatorname{ord}_{M_{i}}(\pi(\alpha))$ lies in $\Lambda_{i}^{+} \backslash\{0\}$. Hence, $\operatorname{ord}_{M_{i}}\left(\pi_{i}\left(\alpha^{A}\right)\right)$ must also belong to $\Lambda_{i}^{+} \backslash\{0\}$. Thus, by [9, Proposition 8.1.4], for any $k_{1}, k_{2} \in I_{p}^{2}$ (in particular for any $\left.k_{1}, k_{2} \in I_{p}\right), \pi_{i}\left(k_{1} \alpha^{A} k_{2}\right.$ ) generates an unbounded subgroup of $G_{i}\left(\mathbb{Q}_{p}\right)$. This is the third condition of Theorem 7.1.

Finally, from the previous discussion we have

$$
\begin{aligned}
& {\left[I_{p}: I_{p} \cap \alpha^{A} I_{p} \alpha^{-A}\right]=\left[I_{p}: I_{p} \cap \alpha^{A} K_{p} \alpha^{-A}\right] \cdot\left[I_{p} \cap \alpha^{A} K_{p} \alpha^{-A}: I_{p} \cap \alpha^{A} I_{p} \alpha^{-A}\right]} \\
& \leq\left[K_{p}: K_{p} \cap \alpha^{A} K_{p} \alpha^{-A}\right] \cdot\left[K_{p}: I_{p}\right] \\
& \leq\left[K_{p}: K_{p} \cap \alpha^{A} G_{\mathbb{Z}_{p}}\left(\mathbb{Z}_{p}\right) \alpha^{-A}\right] \cdot\left[K_{p}: I_{p}\right] \\
& \leq p^{k^{\prime}+f}:=p^{k} \text {. }
\end{aligned}
$$

This is the fourth condition of Theorem 7.1.

\section{The geometric criterion}

In this section, we explain the procedure via which we replace strongly special subvarieties with higher dimensional, strongly special subvarieties given the existence of suitable Hecke correspondences:

Theorem 8.1. Let $(G, X)$ be a Shimura datum and let $K \subset G\left(\mathbb{A}_{f}\right)$ be a neat compact open subgroup, the product of compact open subgroups $K_{p} \subset G\left(\mathbb{Q}_{p}\right)$. Let $X^{+}$be a connected component of $X$ and let $V$ be a special subvariety of $S_{K}(G, X)$. Suppose that $V$ is properly contained in a Hodge generic, irreducible subvariety $Z$ of $S_{K}(G, X)$ and assume that there exist a prime $p$ and an $\alpha \in G\left(\mathbb{Q}_{p}\right)$ such that

- $Z \subset T_{\alpha}(Z)$,

- for every $k_{1}, k_{2} \in K_{p}$, the element $k_{1} \alpha k_{2}$ generates an unbounded subgroup of $G_{i}\left(\mathbb{Q}_{p}\right)$ for each $i$.

Then $Z$ contains a special subvariety $V^{\prime}$ containing $V$ properly. Moreover, if $V$ is strongly special, then $V^{\prime}$ is strongly special.

This theorem is very similar to [9, Theorem 7.2.1] and the proof here is nearly a carbon copy of the proof found in there. Our situation is slightly simplified by the fact that $Z$ is geometrically irreducible. Ensuring that $V^{\prime}$ properly contains $V$ is where we require the stronger condition on $\alpha$. 
Lemma 8.2. If the conclusion of Theorem 8.1 holds for all Shimura data $(G, X)$ with $G$ semisimple of adjoint type, then it holds for all Shimura data.

Proof. Consider the situation in Theorem 8.1. We have a finite morphism of Shimura varieties

$$
f: \operatorname{Sh}_{K}(G, X) \rightarrow \operatorname{Sh}_{K_{\text {ad }}}\left(G^{\mathrm{ad}}, X_{\mathrm{ad}}\right) .
$$

Let $Z_{\text {ad }}$ be the image of $Z$ under this morphism. Similarly, let $V_{\text {ad }}$ be the image of $V$. Thus, $V_{\text {ad }}$ is a special subvariety of $S_{K_{\text {ad }}}\left(G^{\mathrm{ad}}, X_{\mathrm{ad}}\right)$.

Let $\alpha_{\text {ad }}$ denote the image of $\alpha$ in $G^{\text {ad }}\left(\mathbb{Q}_{p}\right)$. The inclusion $Z \subset T_{\alpha}(Z)$ implies that

$$
Z_{\mathrm{ad}} \subset T_{\alpha_{\mathrm{ad}}}\left(Z_{\mathrm{ad}}\right) .
$$

As $K_{\text {ad }}$ is a product of compact open subgroups $K_{\mathrm{ad}, p} \subset G^{\mathrm{ad}}\left(\mathbb{Q}_{p}\right)$, the second condition of Theorem 8.1 implies the analogous condition for $\alpha_{\mathrm{ad}}$ and $K_{\mathrm{ad}, p}$.

As irreducible components of the preimage of a special subvariety by a finite morphism of Shimura varieties are special, it is enough to show that $Z_{\text {ad }}$ contains a special subvariety $V_{\text {ad }}^{\prime}$ containing $V_{\text {ad }}$ properly.

Thus, in this section, we henceforth assume that $G$ is semisimple of adjoint type. We fix a $\mathbb{Z}$-structure on $G$ by choosing a finitely generated free $\mathbb{Z}$-module $W$, choosing a faithful representation

$$
\xi: G \hookrightarrow \mathrm{GL}\left(W_{\mathbb{Q}}\right)
$$

and taking the Zariski closure of $G$ in GL(W). We may choose $\xi$ in such a way that $K$ is contained in $\operatorname{GL}\left(W_{\widehat{\mathbb{Z}}}\right)$. This canonically induces a $\mathbb{Z}$-variation of Hodge structures $\mathcal{F}$ on $\operatorname{Sh}_{K}(G, X)$ and, in particular, on $S_{K}(G, X)$ (see [8, Section 3.2]).

Let $z$ be a Hodge generic point of the smooth locus $Z^{\mathrm{sm}}$ of $Z$. Let $\pi_{1}\left(Z^{\mathrm{sm}}, z\right)$ be the topological fundamental group of $Z^{\mathrm{sm}}$ at the point $z$. We choose a point $x \in X$ lying above $z$. This choice canonically identifies the fibre at $z$ of the locally constant sheaf underlying $\mathcal{F}$ with the $\mathbb{Z}$-module $W$. The action of $\pi_{1}\left(Z^{\mathrm{sm}}, z\right)$ on this fibre is described by the monodromy representation

$$
\rho: \pi_{1}\left(Z^{\mathrm{sm}}, z\right) \rightarrow \pi_{1}\left(S_{K}(G, X), z\right)=G(\mathbb{Q})_{+} \cap K \hookrightarrow \mathrm{GL}(W) .
$$

Since $Z$ is Hodge generic in $\operatorname{Sh}_{K}(G, X)$, the Mumford-Tate group of $\mathcal{F}_{\mid} Z^{\text {sm }}$ at $z$ is $G$. Thus, by [12, Section 1.4], given that the group $G$ is adjoint, the group $\rho\left(\pi_{1}\left(Z^{\mathrm{sm}}, z\right)\right)$ is Zariski dense in $G$. Having fixed a prime $p$ (as in Theorem 8.1), [9, Proposition 4.2.1] implies that the $p$-adic closure of $\rho\left(\pi_{1}\left(Z^{\mathrm{sm}}, z\right)\right)$ in $G\left(\mathbb{Z}_{p}\right)$ is a compact open subgroup $K_{p}^{\prime} \subset K_{p}$.

We have a Galois, pro-étale cover

$$
\pi_{K_{p}}: \operatorname{Sh}_{K^{p}}(G, X) \rightarrow \operatorname{Sh}_{K}(G, X),
$$

with group $K_{p}$, as defined in [9, Section 4.1.3]. Let $\widetilde{Z}$ be an irreducible component of the preimage of $Z$ in $\operatorname{Sh}_{K} p(G, X)$ and let $\widetilde{V}$ be an irreducible component of the preimage of $V$ in $\widetilde{Z}$. By [9, Lemma 7.2.3], we have

Lemma 8.3. The variety $\widetilde{Z}$ is stabilised by the group $K_{p}^{\prime}$ and the set of irreducible components of $\pi_{K_{p}}^{-1}(Z)$ is naturally identified with the finite set $K_{p} / K_{p}^{\prime}$. 
The inclusion $Z \subset T_{\alpha}(Z)$ implies that $\widetilde{Z}$ is an irreducible component of $\pi_{K_{p}}^{-1}\left(T_{\alpha}(Z)\right)$. However, these components are of the form $\widetilde{Z} \cdot k_{1} \alpha k_{2}$ for $k_{1}, k_{2} \in K_{p}$. Therefore, there exist $k_{1}, k_{2} \in K_{p}$ such that $\widetilde{Z}=\widetilde{Z} \cdot k_{1} \alpha k_{2}$.

Corollary 8.4. Let $U_{p}$ be the group generated by $K_{p}^{\prime}$ and $k_{1} \alpha k_{2}$. The variety $\widetilde{Z}$ is stabilised by the group $U_{p}$.

We now conclude the proof of Theorem 8.1. Again, let $\pi_{i}: G \rightarrow G_{i}$ denote the natural morphisms. By the condition placed on $\alpha$, the group $\pi_{i}\left(U_{p}\right)$ is unbounded in $G_{i}\left(\mathbb{Q}_{p}\right)$ for all $i$. Let $G_{1, \mathbb{Q}_{p}}=\prod_{i} H_{i}$ be the decomposition of $G_{1, \mathbb{Q}_{p}}$ into simple factors. Up to renumbering, we can assume that the projection of $U_{p}$ to $H_{1}\left(\mathbb{Q}_{p}\right)$ is unbounded in $H_{1}\left(\mathbb{Q}_{p}\right)$. Let

$$
\tau: \widetilde{H}_{1} \rightarrow H_{1}
$$

be the universal cover of $H_{1}$. We have ([9, Lemma 7.2.6]):

Lemma 8.5. The group $U_{p} \cap H_{1}\left(\mathbb{Q}_{p}\right)$ contains the group $\tau\left(\widetilde{H}_{1}\left(\mathbb{Q}_{p}\right)\right)$ with finite index.

Let $K_{p, 1}$ be the compact open subgroup $\pi_{1}\left(K_{p}\right)$ of $G_{1, \mathbb{Q}_{p}}$ and let $K_{p,>1}$ be the projection of $K$ to

$$
G_{>1, \mathbb{Q}_{p}}:=\prod_{i>1} G_{i, \mathbb{Q}_{p}} .
$$

As $U_{p}$ is an open subgroup of $G\left(\mathbb{Q}_{p}\right)$, it contains a compact open subgroup of $G_{1, \mathbb{Q}_{p}}$ and, in particular, a compact open subgroup $U_{p, 1}$ of $K_{p, 1} \cap \prod_{i>1} H_{i}\left(\mathbb{Q}_{p}\right)$. Similarly, $U_{p}$ contains a compact open subgroup $U_{p,>1}$ of $K_{p,>1}$. By the previous lemma, $U_{p}$ contains the unbounded subgroup $\tau\left(\widetilde{H}_{1}\left(\mathbb{Q}_{p}\right)\right) \cdot U_{p, 1} \cdot U_{p,>1}$. We make the definition ([9, Definition 7.2.7]):

Definition 8.6. We replace $U_{p}$ by its subgroup $\tau\left(\widetilde{H}_{1}\left(\mathbb{Q}_{p}\right)\right) \cdot U_{p, 1} \cdot U_{p,>1}$. We denote by $V^{\prime}$ the Zariski closure of $\pi_{K_{p}}\left(\widetilde{V} \cdot U_{p}\right)$.

Since $\widetilde{Z}$ is stabilised by the group $U_{p}$, the variety $V^{\prime}$ is a subvariety of $Z$. Therefore, let $K_{i}:=\pi_{i}(K)$ and let $\mathcal{K}$ be the neat compact open subgroup $\prod_{i} K_{i}$. We have the natural finite morphism

$$
f: \operatorname{Sh}_{K}(G, X) \rightarrow \operatorname{Sh}_{\mathcal{K}}(G, X)
$$

of Shimura varieties and we let $\mathcal{V}^{\prime}:=f\left(V^{\prime}\right)$ and $\mathcal{V}:=f(V)$. The proof of [9, Lemma 7.2.8] demonstrates that

$$
\mathcal{V}^{\prime}=S_{K_{1}}\left(G_{1}, X_{1}\right) \times \mathcal{V}^{\prime}>1,
$$

where $\mathcal{V}^{\prime}>1$ is the special subvariety of $\prod_{i>1} S_{K_{i}}\left(G_{i}, X_{i}\right)$ given by the projection of $\mathcal{V}^{\prime}$. Hence, $\mathcal{V}^{\prime}$ is a strongly special subvariety of $S_{\mathcal{K}}(G, X)$ and, therefore, since $f$ is a finite morphism of Shimura varieties, $V^{\prime}$ is a strongly special subvariety of $S_{K}(G, X)$. Furthermore, after possibly renumbering the $G_{i}$ (which we are free to do due to the condition placed on $\alpha$ ), we may assume that $\mathcal{V}^{\prime}$ properly contains $\mathcal{V}$. Therefore, $V$ is properly contained in $V^{\prime}$, which concludes the proof of Theorem 8.1. 


\section{Proof of Theorem 1.3}

In this section we will prove Theorem 1.3. In fact, we will prove the following, equivalent statement:

Theorem 9.1. Let $S$ be a Shimura variety and let $\Sigma$ be a set of strongly special subvarieties contained in $S$. Let $Z$ be an irreducible component of the Zariski closure of $\Sigma$ in $S$. Then $Z$ is a strongly special subvariety of $S$.

Lemma 9.2. Theorem 9.1 is equivalent to Theorem 1.3.

Proof. Consider the situation described in Theorem 9.1. If we assume that Theorem 1.3 holds, then there exists a finite set $\left\{V_{1}, \ldots, V_{k}\right\}$ of strongly special subvarieties contained in $Z$ such that, for every $V \in \Sigma, V$ is contained in one of the $V_{i}$. Therefore, $\Sigma$ is contained in the union of the $V_{i}$, which is itself contained in $Z$. Since $Z$ is an irreducible component of the Zariski closure of $\Sigma$, it must be equal to one of the $V_{i}$, proving Theorem 9.1.

Now consider the situation described in Theorem 1.3 and consider the set $\Sigma$ of all strongly special subvarieties of $S$ contained in $Z$. If we assume that Theorem 9.1 holds, the Zariski closure of $\Sigma$ is a union of finitely many strongly special subvarieties $V_{1}, \ldots, V_{k}$. Thus, any strongly special subvariety contained in $Z$ is contained in one of the $V_{i}$, proving Theorem 1.3.

Note that, in order to prove Theorem 9.1, we may assume that the elements of $\Sigma$ are of equal dimension. We first prove Theorem 9.3, following the proof of [9, Theorem 9.2.1]:

Theorem 9.3. Let $\left(G^{\prime}, X^{\prime}\right)$ be a Shimura datum such that $G^{\prime}=G^{\text {ad }}$ and fix a faithful representation

$$
\rho: G^{\prime} \hookrightarrow \mathrm{GL}_{n} .
$$

Let $K^{\prime}$ be a neat compact open subgroup of $G^{\prime}\left(\mathbb{A}_{f}\right)$, equal to a product of compact open subgroups $K_{p}^{\prime} \subset G^{\prime}\left(\mathbb{Q}_{p}\right)$, such that $K^{\prime} \subset \mathrm{GL}_{n}(\widehat{\mathbb{Z}})$. Let $k$ and $f$ be the positive integers given by Theorem 7.1.

Let $\Sigma$ be a set of strongly special subvarieties contained in $S_{K^{\prime}}\left(G^{\prime}, X^{\prime}\right)$. Assume that the elements of $\Sigma$ are of equal dimensiond and that the Zariski closure $Z$ of $\Sigma$ is irreducible. For each $V \in \Sigma$, let $\left(H_{V}, X_{V}\right)$ be the Shimura subdatum defining $V$ and put $\Pi_{V}:=\Pi\left(H_{V}, K_{H}\right)$.

Let $(G, X)$ be a Shimura subdatum of $\left(G^{\prime}, X^{\prime}\right)$ such that $Z$ is contained and Hodge generic in $S_{K}(G, X)$, where $K:=K^{\prime} \cap G\left(\mathbb{A}_{f}\right)$. Let $r:=\operatorname{dim} Z-d>0$ and make one of the following assumptions:

- The $\Pi_{V}$ are bounded as $V$ ranges through $\Sigma$.

- For each $V \in \Sigma$, there exists a prime p not dividing $\Pi_{V}$ such that $K_{p}^{\prime}=G_{\mathbb{Z}_{p}}^{\prime}\left(\mathbb{Z}_{p}\right)$ and

$$
p^{(k+2 f) \cdot 2^{r}} \cdot\left(\operatorname{deg}_{\mathscr{L}_{K}} Z\right)^{2^{r}}<c \cdot \Pi_{V}^{\delta} .
$$

Then, for each $V \in \Sigma, Z$ contains a strongly special subvariety of $S_{K^{\prime}}\left(G^{\prime}, X^{\prime}\right)$ containing $V$ properly.

In this section, we will use the term uniform to mean depending only on $\left(G^{\prime}, X^{\prime}\right), K^{\prime}$ and $\rho$. 
Proof. Firstly, we consider the case that, as $V$ ranges through $\Sigma, \Pi_{V}$ is bounded. That is to say, the primes dividing any given $\Pi_{V}$ belong to a fixed, finite set, whose product we denote $\Pi$.

By Theorem 7.1, for any prime $p$ not dividing $\Pi$ such that $K_{p}^{\prime}=G_{\mathbb{Z}_{p}}^{\prime}\left(\mathbb{Z}_{p}\right)$, there exists a compact open subgroup

$$
I_{p} \subset K_{p}:=K_{p}^{\prime} \cap G\left(\mathbb{Q}_{p}\right)=G_{\mathbb{Z}_{p}}\left(\mathbb{Z}_{p}\right)
$$

and an element $\alpha \in G\left(\mathbb{Q}_{p}\right)$ satisfying the four requirements of Theorem 7.1, for each $V \in \Sigma$. However, in this case we will choose these objects slightly more precisely: recall that, by Lemma 7.3, for each $V \in \Sigma$, there exists a non-trivial, maximal, split torus $S_{V} \subset H_{V, \mathbb{Q}_{p}}$ such that $S_{V, \mathbb{Z}_{p}}$ is a torus. Since $S_{V}$ is split, it is conjugate via an element of $\mathrm{GL}_{n}\left(\mathbb{Q}_{p}\right)$ to a subtorus of the diagonal matrices. By Lemma 7.4, after possibly replacing $\boldsymbol{\Sigma}$ by a Zariski dense subset, we may assume that this torus is fixed, i.e. that the $S_{V}$ are all conjugate by elements of $\mathrm{GL}_{n}\left(\mathbb{Q}_{p}\right)$ to a fixed torus $S:=S_{V_{0}}$ for some $V_{0} \in \Sigma$. Let $I_{p} \subset K_{p}$ and $\alpha \in G\left(\mathbb{Q}_{p}\right)$ be the objects given by Theorem 7.1 applied to $V_{0}$.

Now consider another $V \in \Sigma$ and let $g \in \mathrm{GL}_{n}\left(\mathbb{Q}_{p}\right)$ be such that $g S_{V} g^{-1}=S$. Since $S_{V, \mathbb{Z}_{p}}$ is a torus, $S$ stabilises the lattice $g \mathbb{Z}_{p}^{n}$. Therefore, by [8, Lemma 3.3.1], since $S_{\mathbb{Z}_{p}}$ is a torus, there exists an element $c \in Z_{G}(S)\left(\mathbb{Q}_{p}\right)$ such that $g \mathbb{Z}_{p}^{n}=c \mathbb{Z}_{p}^{n}$, where $Z_{G}(S)$ is the centraliser of $S$ in $G$. Therefore, there exists an element $k \in \mathrm{GL}_{n}\left(\mathbb{Z}_{p}\right)$ such that $g=c k$ and so the $S_{V}$ are all conjugate by elements of $\mathrm{GL}_{n}\left(\mathbb{Z}_{p}\right)$. If we further assume that $p$ is a prime such that $G_{\mathbb{F}_{p}}$ is smooth, the final paragraph of the proof of [8, Proposition 7.3.1] explains that, again, after possibly replacing $\boldsymbol{\Sigma}$ by a Zariski dense subset, we may assume that the $S_{V}$ are all conjugate by elements of $K_{p}$ and, therefore, by elements of $I_{p}$.

Therefore, for each $V \in \Sigma$, we let $g_{V} \in I_{p}$ be such that $S_{V}=g_{V} S g_{V}^{-1}$. It follows that $I_{p}$ and $\alpha_{V}:=g_{V} \alpha g_{V}^{-1}$ satisfy the requirements of Theorem 7.1 applied to $V$. Furthermore, if we let $I \subset K$ be the compact open subgroup $K^{p} I_{p} \subset G\left(\mathbb{A}_{f}\right)$, then the Hecke correspondences $T_{\alpha_{V}}$ on $\operatorname{Sh}_{I}(G, X)$ all coincide with $T_{\alpha}$.

Let

$$
\tau: \operatorname{Sh}_{I}(G, X) \rightarrow \operatorname{Sh}_{K}(G, X)
$$

be the induced morphism of Shimura varieties and let $\widetilde{Z}$ be an irreducible component of the preimage $\tau^{-1}(Z)$. For each $V \in \Sigma$, let $\widetilde{V} \subset S_{I}(G, X)$ be an irreducible component of the preimage $\tau^{-1}(V)$ contained in $\widetilde{Z}$. Each $\widetilde{V}$ is a strongly special subvariety of $S_{I}(G, X)$ defined by the Shimura subdatum $\left(H_{V}, X_{V}\right)$. Denote the set of the $\widetilde{V}$ by $\widetilde{\Sigma}$. By the second requirement of Theorem 7.1, we have $\widetilde{V} \subset T_{\alpha}(\widetilde{V})$ for every $\widetilde{V} \in \widetilde{\Sigma}$. Hence, $\widetilde{\Sigma}$ is contained in $\widetilde{Z} \cap T_{\alpha}(\widetilde{Z})$ and, therefore, $\widetilde{Z} \subset T_{\alpha}(\widetilde{Z})$.

As $\alpha$ satisfies the third requirement of Theorem 7.1, we can apply Theorem 8.1 to this $\alpha$ and conclude that, for each $\widetilde{V} \in \widetilde{\Sigma}$, there exists a special subvariety $\widetilde{V}^{\prime} \subset \widetilde{Z}$ containing $\widetilde{V}$ properly whose image in $\operatorname{Sh}_{K^{\prime}}\left(G^{\prime}, X^{\prime}\right)$ is strongly special. As $\tau$ preserves the property of being special, exhibiting a special subvariety $V^{\prime} \subset Z$ containing $V$ properly is equivalent to exhibiting a special subvariety $\widetilde{V}^{\prime} \subset \widetilde{Z}$ containing $\widetilde{V}$ properly.

Thus, we consider the case that $\Pi_{V}$ is unbounded as $V$ ranges through $\Sigma$. Hence, we may assume that $\Pi_{V}$ is larger than any uniform constant. We proceed by induction on $r$. Consider first the case $r=1$ and let $V \in \Sigma$.

By the second assumption of Theorem 9.3, there exists a compact open subgroup $I_{p} \subset K_{p}$ and an element $\alpha \in G\left(\mathbb{Q}_{p}\right)$ satisfying the four requirements of Theorem 7.1 applied to $V$. 
Let $I \subset K$ be the compact open subgroup $K^{p} I_{p} \subset G\left(\mathbb{A}_{f}\right)$ and let

$$
\tau: \operatorname{Sh}_{I}(G, X) \rightarrow \operatorname{Sh}_{K}(G, X)
$$

be the induced morphism of Shimura varieties. It follows from the first requirement of Theorem 7.1 that the degree of $\tau$ is bounded above by $p^{f}$.

Let $\widetilde{V} \subset S_{I}(G, X)$ be an irreducible component of the preimage $\tau^{-1}(V)$. It is a strongly special subvariety of $S_{I}(G, X)$ defined by the Shimura subdatum $\left(H_{V}, X_{V}\right)$ of $(G, X)$. By the projection formula (see [9, Proposition 5.3.2(1)]) and Theorem 1.4,

$$
\operatorname{deg}_{\mathscr{L}_{I}} \widetilde{V} \geq \operatorname{deg}_{\mathscr{L}_{K}} V>c \cdot \Pi_{V}^{\delta} .
$$

Let $\widetilde{Z}$ be an irreducible component of the preimage $\tau^{-1}(Z)$ containing $\widetilde{V}$. Thus, $\widetilde{Z}$ is Hodge generic in $\operatorname{Sh}_{I}(G, X)$ and

$$
\operatorname{deg}_{\mathscr{L}_{I}} \widetilde{Z} \leq p^{f} \cdot d_{Z}
$$

As $\tau$ preserves the property of being special, exhibiting a special subvariety $V^{\prime} \subset Z$ containing $V$ properly is equivalent to exhibiting a special subvariety $\widetilde{V}^{\prime} \subset \widetilde{Z}$ containing $\widetilde{V}$ properly.

By the second requirement of Theorem 7.1, we have $\widetilde{V} \subset T_{\alpha}(\widetilde{V})$. Hence, $\widetilde{V} \subset \widetilde{Z} \cap T_{\alpha}(\widetilde{Z})$. Given their dimensions, if $\widetilde{Z}$ and $T_{\alpha}(\widetilde{Z})$ intersect properly, then $\widetilde{V}$ is an irreducible component of the intersection. Thus,

$$
\begin{aligned}
c \cdot \Pi_{V}^{\delta}<\operatorname{deg}_{\mathscr{L}_{I}} \widetilde{V} & \leq \operatorname{deg}_{\mathscr{L}_{I}}\left(\widetilde{Z} \cap T_{\alpha}(\widetilde{Z})\right) \\
& \leq\left(\operatorname{deg}_{\mathscr{L}_{I}} \widetilde{Z}\right)^{2} \cdot\left[I_{p}: I_{p} \cap \alpha I_{p} \alpha^{-1}\right] \\
& <p^{k+2 f} \cdot d_{Z}^{2},
\end{aligned}
$$

contradicting the second assumption of the theorem. Therefore, the intersection cannot be proper. Thus, $\widetilde{Z} \subset T_{\alpha}(\widetilde{Z})$ and, since $\alpha$ satisfies the second condition of Theorem 8.1 , there exists a special subvariety $\widetilde{V^{\prime}} \subset \widetilde{Z}$ containing $\widetilde{V}$ properly whose image in $\operatorname{Sh}_{K^{\prime}}\left(G^{\prime}, X^{\prime}\right)$ is strongly special.

Therefore, we consider the case $r>1$. Suppose that the conclusion of Theorem 9.3 holds for all subvarieties $V$ and $Z$ of $\operatorname{Sh}_{K}(G, X)$ as in the statement of Theorem 9.3 such that $0<\operatorname{dim} Z-d<r$ and consider the case that $\operatorname{dim} Z=d+r$. We have $\widetilde{V}, \widetilde{Z}$, a compact open subgroup $I \subset K$ and an $\alpha \in G\left(\mathbb{Q}_{p}\right)$, constructed as in the case $r=1$, where

$$
\operatorname{deg}_{\mathscr{L}_{I}} \widetilde{V}>c \cdot \Pi_{V}^{\delta} \quad \text { and } \quad \operatorname{deg}_{\mathscr{L}_{I}} \widetilde{Z} \leq p^{f} \cdot d_{Z}
$$

Suppose that $\widetilde{Z} \subset T_{\alpha}(\widetilde{Z})$. In this case we can apply Theorem 8.1 to deduce that there exists a special subvariety $\widetilde{V}^{\prime} \subset \widetilde{Z}$ containing $\widetilde{V}$ properly whose image in $\operatorname{Sh}_{K^{\prime}}\left(G^{\prime}, X^{\prime}\right)$ is strongly special.

Therefore, suppose that the intersection $\widetilde{Z} \cap T_{\alpha}(\widetilde{Z})$ is proper. By the second requirement of Theorem 7.1, $\widetilde{V} \subset \widetilde{Z} \cap T_{\alpha}(\widetilde{Z})$. Choose an irreducible component $\widetilde{Y} \subset S_{I}(G, X)$ of $\widetilde{Z} \cap T_{\alpha}(\widetilde{Z})$ containing $\widetilde{V}$ and denote its image in $\operatorname{Sh}_{K}(G, X)$ by $Y$. Thus, $Y$ is irreducible and satisfies $r_{Y}:=\operatorname{dim} Y-d<r$. To show that $r_{Y}>0$ it suffices to check that $\widetilde{V}$ is not a component of $\widetilde{Z} \cap T_{\alpha}(\widetilde{Z})$. However, if this were true, we would have

$$
c \cdot \Pi_{V}^{\delta}<p^{k+2 f} \cdot d_{Z}^{2}
$$

as in the case $r=1$, contradicting the second assumption of Theorem 9.3. 
Let $\left(P, X_{P}\right)$ be a Shimura datum of $(G, X)$, defining the smallest special subvariety of $S_{I}(G, X)$ containing $\widetilde{Y}$. Let $X_{P}^{+} \subset X^{+}$be the corresponding connected component of $X_{P}$. Define $K_{P}:=K \cap P\left(\mathbb{A}_{F}\right)$ and $I_{P}:=I \cap P\left(\mathbb{A}_{f}\right)$. We have the commutative diagram

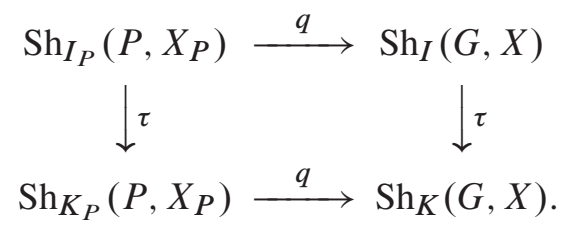

Let $\widetilde{V}_{P}$ be an irreducible component of $q^{-1}(\widetilde{V})$ contained in $S_{I_{P}}\left(P, X_{P}\right)$; let $V_{P}:=\tau\left(\widetilde{V}_{P}\right)$.

Let $\widetilde{Y}_{P} \subset S_{I_{P}}\left(P, X_{P}\right)$ be an irreducible component of $q^{-1}(\widetilde{Y})$ containing $\widetilde{V}_{P}$. In particular, $\widetilde{Y}_{P}$ is a Hodge generic subvariety of $S_{I_{P}}\left(P, X_{P}\right)$. Define $Y_{P}:=\tau\left(\widetilde{Y}_{P}\right)$, a Hodge generic subvariety of $S_{K_{P}}\left(P, X_{P}\right)$.

We have

$$
\operatorname{deg}_{\mathscr{L}_{K_{P}}} Y_{P} \leq \operatorname{deg}_{\mathscr{L}_{I_{P}}} \widetilde{Y}_{P} \leq \operatorname{deg}_{\mathscr{L}_{I}} \widetilde{Y} \leq \operatorname{deg}_{\mathscr{L}_{I}}\left(\widetilde{Z} \cap T_{\alpha}(\widetilde{Z})\right)<p^{k+2 f} \cdot d_{Z}^{2},
$$

where the first inequality comes from the projection formula, the second comes from [9, Proposition 5.3.10], the third is due to the fact that $\widetilde{Y}$ is an irreducible component of $\widetilde{Z} \cap T_{\alpha}(\widetilde{Z})$, and the last inequality was demonstrated previously.

Lemma 9.4. The data $P, X_{P}, X_{P}^{+}, K_{P}, V_{P}$ and $Y_{P}$ satisfy the conditions of Theorem 9.3 (in place of $G, X, X^{+}, K, V$ and $Z$, respectively).

Proof. Firstly, note that the image of $V_{P}$ in $\operatorname{Sh}_{K^{\prime}}\left(G^{\prime}, X^{\prime}\right)$ is strongly special since it is still defined by the Shimura datum $\left(H_{V}, X_{V}\right)$. Let

$$
r_{P}:=\operatorname{dim} Y_{P}-\operatorname{dim} V_{P} .
$$

Thus, $r_{P}=r_{Y}>0$. We must verify that $P, X_{P}, X_{P}^{+}, K_{P}, V_{P}$ and $Y_{P}$ satisfy the second condition of Theorem 9.3 for the same prime $p$.

From the above inequalities we have

$$
p^{(k+2 f) \cdot 2^{r_{P}}} \cdot\left(\operatorname{deg}_{\mathscr{L}_{K_{P}}} Y_{P}\right)^{2^{r} P} \leq p^{(k+2 f) \cdot 2^{r}+1} \cdot d_{Z}^{2^{r_{P}+1}}
$$

and, as $r_{P}+1 \leq r$, we deduce from the second assumption of Theorem 9.3 that

$$
p^{(k+2 f) \cdot 2^{r_{P}}} \cdot\left(\operatorname{deg}_{\mathscr{L}_{K_{P}}} Y_{P}\right)^{2^{r_{P}}}<c \cdot \Pi_{V}^{\delta} .
$$

As $r_{P}<r$, by the induction hypothesis, we can apply Theorem 9.3 to $P, X_{P}, X_{P}^{+}, K_{P}$, $V_{P}$ and $Y_{P}$. Thus $Y_{P}$ contains a special subvariety $V_{P}^{\prime}$, which contains $V_{P}$ properly and whose image in $\operatorname{Sh}_{K^{\prime}}\left(G^{\prime}, X^{\prime}\right)$ is strongly special. This implies that $Z$ contains a special subvariety $V^{\prime}$, which contains $V$ properly and whose image in $\operatorname{Sh}_{K^{\prime}}\left(G^{\prime}, X^{\prime}\right)$ is strongly special.

Therefore, in order to prove Theorem 9.1, it suffices to prove the following lemma:

Lemma 9.5. Let $V \in \Sigma$. There exists a uniform constant $c_{11}$ such that, if $\Pi_{V}>c_{11}$, then there exists a prime $p$ not dividing $\Pi_{V}$ such that $K_{p}^{\prime}=G_{\mathbb{Z}_{p}}^{\prime}\left(\mathbb{Z}_{p}\right)$ and

$$
p^{(k+2 f) \cdot 2^{r}} \cdot\left(\operatorname{deg}_{\mathscr{L}_{K}} Z\right)^{2^{r}}<c \cdot \Pi_{V}^{\delta} .
$$


Proof. By a theorem of Chebyshev, there exist absolute positive constants $c_{12}$ and $c_{13}$ such that the number of primes $\pi(x)$ less than a given real number $x \geq 2$ is bounded below by $c_{12} \frac{x}{\log x}$ and above by $c_{13} \frac{x}{\log x}$. Therefore, for any fixed $\gamma>\epsilon>0$,

$$
\pi\left(\Pi_{V}^{\gamma}\right) \gg \frac{\Pi_{V}^{\gamma}}{\log \Pi_{V}^{\gamma}} \gg \Pi_{V}^{\gamma-\epsilon} .
$$

If we denote by $\omega\left(\Pi_{V}\right)$ the number of primes dividing $\Pi_{V}$, we have the trivial estimate

$$
\omega\left(\Pi_{V}\right) \leq \frac{\log \Pi_{V}}{\log 2} \ll \Pi_{V}^{\epsilon}
$$

Note that $K_{p}^{\prime}=G_{\mathbb{Z}_{p}}^{\prime}\left(\mathbb{Z}_{p}\right)$ holds for all primes $p$ greater than a uniform constant. Therefore, if we set

$$
\gamma=\frac{\delta}{(k+2 f) 2^{r}}-\epsilon>2 \epsilon>0
$$

provided $\Pi_{V}$ is larger than a uniform constant, we can find a prime $p$ satisfying the requirements of the lemma.

\section{The André-Oort conjecture}

We will prove the following theorem, which appears as [9, Theorem 1.2.2]. The difference between our proof and the one appearing there is that ours does not depend on any results from ergodic theory.

Theorem 10.1. Let $(G, X)$ be a Shimura datum and let $K$ be a compact open subgroup of $G\left(\mathbb{A}_{f}\right)$. Let $\Sigma$ be a set of special subvarieties in $\operatorname{Sh}_{K}(G, X)$ and let $Z$ be an irreducible component of the Zariski closure of $\Sigma$ in $\operatorname{Sh}_{K}(G, X)$. We make one of the following assumptions:

- Assume the generalised Riemann hypothesis for CM fields.

- Assume that there exists a faithful representation $G \hookrightarrow \mathrm{GL}_{n}$ such that, with respect to this representation, the generic Mumford-Tate groups $\mathrm{MT}_{V}$ of the $V \in \Sigma$ lie in one $\mathrm{GL}_{n}(\mathbb{Q})$-conjugacy class.

Then $Z$ is a special subvariety of $\operatorname{Sh}_{K}(G, X)$.

Proof. Fix a connected component $X^{+}$of $X$. We may assume that $Z$ lies in the connected component $S_{K}(G, X)$. Now, [9, Theorem 2.5.3] produces a dichotomy: either the subvarieties $V$ have Galois orbits whose degrees are bounded from below by an invariant unbounded as we range through $\Sigma$ or there exists a finite set $\left\{T_{1}, \ldots, T_{r}\right\}$ of subtori of $G$, anisotropic over $\mathbb{R}$, such that each $V \in \Sigma$ is $T_{i}$-special for some $i \in\{1, \ldots, r\}$ (see [19, Definition 3.1 and Definition 3.2] for the definition of $T$-special).

If the former occurs then [9, Theorem 3.2.1] implies Theorem 10.1. Otherwise, we may assume that every $V \in \Sigma$ is $T$-special for some fixed subtorus $T$ of $G$ such that $T_{\mathbb{R}}$ is anisotropic. Thus, by [19, Lemma 3.3 and Lemma 3.5], there exist $q \in G(\mathbb{Q}), \theta \in G\left(\mathbb{A}_{f}\right)$ and, for each $V \in \Sigma$, a $q T q^{-1}$-Shimura subdatum $\left(H_{V}, X_{V}\right)$ of $(G, X)$, where $H_{V}$ is the 
generic Mumford-Tate group of $X_{V}$, such that $V$ is the image of $X_{V}^{+} \times\{\theta\}$ in $S_{K}(G, X)$ (see [19, Definition 3.1] for the definition of a $T$-Shimura subdatum). Hence, after replacing $Z$ by an irreducible component of its image under a suitable Hecke correspondence, we may assume that each $V$ is a standard $T$-special subvariety of $S_{K}(G, X)$, associated to a $T$-Shimura subdatum $\left(H_{V}, X_{V}\right)$, with $H=\mathrm{MT}\left(X_{V}\right)$ (see [19, Definition 3.2] for the definition of a standard $T$-special subvariety).

Thus, by [19, Lemma 3.6 and Lemma 3.7], for every $V \in \Sigma,\left(H_{V}, X_{V}\right)$ is a Shimura subdatum of a fixed $T$-Shimura subdatum $\left(L, X_{L}\right)$. Therefore, we may assume that $\Sigma$ is contained in $S_{L\left(\mathbb{A}_{f}\right) \cap K}\left(L, X_{L}\right)$. Let $\left(L^{\text {ad }}, X_{L, \text { ad }}\right)$ be the adjoint Shimura datum and let $K_{L}$ be a compact open subgroup of $L^{\text {ad }}\left(\mathbb{A}_{f}\right)$ containing the image of $L\left(\mathbb{A}_{f}\right) \cap K$. Thus, we have an induced morphism of Shimura varieties

$$
f: \operatorname{Sh}_{L\left(\mathbb{A}_{f}\right) \cap K}\left(L, X_{L}\right) \rightarrow \operatorname{Sh}_{K_{L}}\left(L^{\mathrm{ad}}, X_{L, \text { ad }}\right) .
$$

Let $V^{\text {ad }}$ be the image of $V$ under $f$. Since $T$ is the connected centre of $H_{V}$ and $T$ is contained in the centre of $L, V^{\text {ad }}$ is defined by a Shimura subdatum $\left(H_{V}^{\prime}, X_{V}^{\prime}\right)$ of $\left(L^{\text {ad }}, X_{L^{\text {ad }}}\right)$ such that $H_{V}^{\prime}$ is semisimple. Since, by [8, Proposition 2.2], $Z$ is special if and only if its image under $f$ is special, we have reduced Theorem 10.1 to Theorem 1.3.

Acknowledgement. The author is deeply indebted to Andrei Yafaev, who has been beyond reproach in his role as a supervisor. He is also grateful to the Department of Mathematics at University College London and to the organisers and sponsors of the "Around the Zilber-Pink conjectures' summer school held in Paris during June/July 2012. He would like to thank the ANR Modig Programme for the opportunity to visit Emmanuel Ullmo at the IHES in October 2013, whose comments have been extremely helpful. Finally, the author would like to thank the referee, not only for his technical remarks, but also for his comments regarding the overall presentation and for his attention to detail.

\section{References}

[1] A. Borel and J. Tits, Groupes réductifs, Publ. Math. Inst. Hautes Études Sci. 27 (1965), 55-151.

[2] F. Bruhat and J. Tits, Groupes réductifs sur un corps local II, Publ. Math. Inst. Hautes Études Sci. 60 (1984), 1-184.

[3] L. Clozel and E. Ullmo, Equidistribution adélique des tores et équidistribution des points CM, Doc. Math. Extra Vol. (2006), 233-260.

[4] L. Clozel and E. Ullmo, Equidistribution de sous-variétés spéciales, Ann. of Math. (2) 161 (2006), 1571-1588.

[5] C. Daw, A simplified proof of the André-Oort conjecture for products of modular curves, Arch. Math. 98 (2012), 433-440.

[6] C. Daw, On torsion of class groups of CM tori, Mathematika 58 (2012), no. 2, 305-318.

[7] M. Demazure and A. Grothendieck, Schémas en groupes. Fasc. 4. Exposés XII à XIV. Séminaire de géométrie algébrique de l'Institut des Hautes Études Scientifiques (1963/64), Institut des Hautes Études Scientifiques, Bures-Sur-Yvette 1964.

[8] S. Edixhoven and A. Yafaev, Subvarieties of Shimura varieties, Ann. of Math. (2) 157 (2003), no. 2, $621-645$.

[9] B. Klingler and A. Yafaev, The André-Oort conjecture, preprint 2012, http://arxiv .org/abs/1209.0936; to appear in Ann. of Math. (2).

[10] G. Margulis, Discrete subgroups of semisimple Lie groups, Ergeb. Math. Grenzgeb. (3) 17, Springer-Verlag, Berlin 1991.

[11] J. Milne, Introduction to Shimura varieties, expository notes 2004, www . jmilne.org/math.

[12] B. Moonen, Linearity properties of Shimura varieties I, J. Algebraic Geom. 7 (1998), 539-567.

[13] D. Mumford, Hirzebruch's proportionality principle in the non-compact case, Invent Math. 42 (1977), 239-277. 
[14] H. Oh, Adelic version of Margulis arithmeticity theorem, Math. Ann. 321 (2001), no. 4, 789-815.

[15] V. Platonov and A. Rapinchuk, Algebraic groups and number theory, Pure Applied Math. 139, Academic Press, Boston 1994.

[16] G. Prasad, Volumes of S-arithmetic quotients of semi-simple groups, Publ. Math. Inst. Hautes Études Sci. 69 (1989), 91-114.

[17] T. Springer, Reductive groups, in: Automorphic forms, representations and L-functions (Corvallis 1977), Proc. Sympos. Pure Math. 33, Part 1, American Mathematical Society, Providence (1979), 3-27.

[18] J. Tits, Reductive groups over local fields, in: Automorphic forms, representations and L-functions (Corvallis 1977), Proc. Sympos. Pure Math. 33, Part 1, American Mathematical Society, Providence (1979), $29-69$.

[19] E. Ullmo and A. Yafaev, Galois Orbits and equidistribution of special subvarieties: Towards the André-Oort conjecture, preprint 2012, http: //arxiv.org/abs/1209.0934; to appear in Ann. of Math. (2).

[20] A. Vasiu, Extension theorems for reductive group schemes, preprint 2012, http://arxiv.org/abs/math/ 0406508.

[21] A. Vasiu, Geometry of Shimura varieties of Hodge type over finite fields, in: Higher-dimensional geometry over finite fields (Göttingen 2007), IOS Press, Amsterdam (2008), 197-243.

[22] W. Waterhouse, Introduction to affine group schemes, Grad. Texts in Math. 66, Springer-Verlag, New York 1979.

[23] A. Yafaev, A conjecture of Yves André, Duke Math. J. 132 (2006), no. 3, 393-407.

Christopher Daw, Department of Mathematics, University College London,

Gower street, WC1E 6BT London, United Kingdom

e-mail: c.daw@ucl.ac.uk

Eingegangen 3. Oktober 2012, in revidierter Fassung 3. Mai 2014 